\title{
A MEASURE ON THE HARMONIC BOUNDARY OF A RIEMANN SURFACE
}

\author{
MITSURU NAKAI
}

\section{Introduction}

1. In the usual theory of harmonic functions on a plane domain, the fact that the boundary of the domain is realized relative to the complex plane plays an essential role and supplies many powerful tools, for instance, the solution of Dirichlet problem. But in the theory of harmonic functions on a general domain, i.e. on a Riemann surface, the main difficulty arises from the lack of the "visual" boundary of the surface. Needless to say, in general we cannot expect to get the "relative" boundary with respect to some other larger surface. In view of this, we need some "abstract" compactification. It seems likely that we cannot expect to get the "universal" boundary which is appropriate for any harmonic functions since there exist many surfaces which do not admit some classes of harmonic functions as the classification theory shows. Hence we need many compactifications corresponding to what class of harmonic functions we are going to investigate.

There are two typical compactifications, as Royden [10] pointed out, Martin's compactification and Royden's compactification. The former seems appropriate for the study of $H B$-functions and the latter aims to be used for the study of $H B D$-functions, which we are going to investigate in this paper. A similar investigation is carried out by Kuramochi in the direction of Martin.

2. Royden's compactification is first introduced by Royden and has been studied by Mori, Mori-Ôta, Kusunoki-Mori and the present author. But these investigations seem to be restricted for $H B D$-functions.

The first aim of the present paper is to show that Royden's boundary is powerful not only for $H B D$-functions but also for $H D$-functions and we shall show that the elementary theory of $H D$-functions can be systematically developed by using the Royden's boundary. The main tool is the "harmonic decomposition".

Received May 23, 1960. 
The second aim is to show that a maximum principle holds for $H B$-functions and $H D$-functions, that is, the range of values of an $H B$ - (or $H D^{-}$) function is determined by the range of values on an arbitrarily small neighborhood of the harmonic boundary (or on the harmonic boundary) which is an essential part of the Royden's boundary.

These are discussed in Chapter 1.

3. Comparing with Martin's boundary, we feel the lack of tools for the investigation of Dirichlet problem with given boundary values on the harmonic boundary. Aiming to supply this, we define a measure on the harmonic boundary which seems very natural. This is an analogy of Poisson's representation of harmonic functions in the unit circle. We shall discuss the elementary properties of this measure or integral representation of harmonic functions in Chapter 2. In the integral representation of some harmonic functions, the boundary behaviour is not solved but in a special case and we believe that this is a very important problem left to be solved.

In the final Chapter 3 , we give a characterization of $H D$-minimality considered by Constantinescu-Cornea as an application of our measure mentioned above.

At the end, we should like to express their warmest thanks to Professor K. Noshiro for his kind guidance and suggestion and also to Professor T. Kuroda for his valuable discussion.

\section{Harmonic boundary and a maximum principle}

1. Royden's algebra $M(R)$. Let $R$ be a Riemann surface. Suppose that a complex-valued function $f(z)$ defined on $R$ satisfies the following three conditions :

(M.1) $f(z)$ is absolutely continuous in the sense of Tonelli ; ${ }^{1)}$

(M.2) $f(z)$ is bounded in the absolute value;

(M.3) Dirichlet integral of $f(z)$ taken over $R$ is finite.

We denote by $M(R)$ the totality of complex-valued functions $f(z)$ defined on $R$

1) A complex-valued function $f(x, y)$ defined on a rectangle $[a, b ; c, d]$ is said to be absolutely continuous in the sense of Tonelli (abreviated as a.c.T) if $f(x, y)$ is absolutely continuous as a function of $x$ for almost every fixed $y$ in $[c, d]$ and the corresponding fact holds if $x$ and $y$ are interchanged and further $\partial f / \partial x$ and $\partial f / \partial y$ are locally integrable in $[a, b ; c, d]$. This notion is carried over Riemann surfaces by using local parameters. 
satisfying the above three conditions. By the usual algebraic operations, $M(R)$ becomes an algebra over the complex number field. We call $M(R)$ Royden's algebra associated with $R$. Royden's algebra $M(R)$ determines the quasi-conformal structure of $R$ (cf. [7]).

We shall use the following norms (or semi-norm) in $M(R)$, i.e.

uniform norm: $\|f\|=\sup _{R}|f(z)|$;

Dirichlet semi-norm: $\quad(D[f])^{1 / 2}=\left(\iint_{R} d f \wedge{ }^{*} \overline{d f}\right)^{1 / 2}$;

$M$-norm : $\|f\|_{M}=\|f\|+(D[f])^{1 / 2}$.

We denote by $M^{1}(R)$ the totality of complex-valued $C^{1}$-functions $f(z)$ defined on $R$ satisfying (M.2) and (M.3). Then $M^{1}(R)$ is an subalgebra of $M(R)$. We have

Lemma 1.1 ([6]). Royden's algebra $M(R)$ is a Banach algebra under the norm $\|f\|_{M}$ and $M^{1}(R)$ is dense in $M(R)$ with respect to this norm.

In virtue of this Lemma, we may freely use Green's formula and the Dirichlet principle for functions in $M(R)$.

We shall use some convergence topologies in $M(R)$ defined by the following: a sequence $\left\{f_{n}(z)\right\}$ of functions in $M(R)$ converges to a function $f(z)$ in $C$-topology if it converges uniformly on any compact subset of $R$. If a sequence $\left\{f_{n}(z)\right\}$ is bounded and converges to $f(z)$ in $C$-topology, we say that $\left\{f_{n}(z)\right\}$ converges to $f(z)$ in $B$-topology. Finally we say that a sequence $\left\{f_{n}(z)\right\}$ converges to $f(z)$ in $C D$ (or $B D$ )-topology if it converges in $C$ (or $B$ )-topology and at the same time in $D$-topology, where $D$-topology is the topology defined by Dirichlet semi-norm. These topologies above mentioned, i.e. $C$-, $B-, D-, C D$ and $B D$-topology are purely algebraic notion in the sense of von Neumann in $M(R)$, since the algebraic structure of $M(R)$ determines (and is determined by) the quasi-conformal structure of $R$, which determines these topologies.

It is proved that $M(R)$ is complete with respect to $B D$-topology ([6]).

We denote by $M_{0}(R)$ the totality of functions in $M(R)$ with compact support and we denote by $M_{\Delta}(R)$ the closure of $M_{0}(R)$ in $M(R)$ with respect to $B D$ topology. Now we have

Lemma 1.2. The set $M_{\Delta}(R)$ is an ideal of $M(R)$.

Proof. It is clear that $M_{\Delta}(R)$ is a submodule of $M(R)$. So we have only 
to prove $g(z) f(z)$ belongs to $M_{\Delta}(R)$ if $g(z)$ is in $M(R)$ and $f(z)$ is in $M_{\Delta}(R)$. We choose a sequence $\left\{f_{n}(z)\right\}$ in $M_{0}(R)$ which converges to $f(z)$ in $B D$-topology. Then the sequence $\left\{g(z) f_{n}(z)\right\}$ is contained in $M_{0}(R)$ and converges to $g(z) f(z)$ in $B$-topology. So we have to prove that the sequence $\left\{g(z) f_{n}(z)\right\}$ converges to $g(z) f(z)$ in $D$-topology. Replacing $f_{n}(z)$ by $f_{n}(z)-f(z)$, we may assume that $\left\{f_{n}(z)\right\}$ converges to 0 in $B D$-topology and we have to prove that $\left\{g(z) f_{n}(z)\right\}$ converges to 0 in $D$-topology. Let $K$ be an arbitrary compact subset of $R$. We have

$$
\begin{aligned}
D\left[g f_{n}\right] & =\iint_{R}\left(g d f_{n}+f_{n} d g\right) \wedge\left(g^{*} d f_{n}+f_{n}^{*} d g\right) \\
& \leqq 2 \iint_{R}|g|^{2} d f_{n} \wedge{ }^{*} d f_{n}+2 \iint_{R}\left|f_{n}\right|^{2} d g \wedge * d g \\
& \leqq 2\|g\|^{2} D\left[f_{n}\right]+2 \sup _{K}\left|f_{n}(z)\right|^{2} D[g]+2\left\|f_{n}\right\|_{\mid}^{2} \iint_{R-K} d g \wedge * d g .
\end{aligned}
$$

From this we get

$$
\lim _{n} D\left[g f_{n}\right] \leqq\left(2 \lim _{n}\left\|f_{n}\right\|^{2}\right) \iint_{R-K} d g \wedge * d g .
$$

Letting $K \uparrow R$, we have the required property. This completes the proof.

2. Royden's compactification $R^{*}$ and the harmonic boundary $\Delta$. Let $R$ be a Riemann surface. Here we give a definition of Royden's compactification of $R$ which is similar to that of Čech's compactification of locally compact spaces. A set $R^{*}$ is said to be Royden's compactification of $R$ if the following two conditions are satisfied:

(R.1) $R^{*}$ is a compact Hausdorff space containing $R$ as its open and dense subset;

(R.2) any function in $M(R)$ can be extended to $R^{*}$ so as to be a continuous function defined on $R^{*}$.

From the general theory of compactifications of Čech type, it is shown that $R^{*}$ always exists and unique up to a homeomorphism and this homeomorphism can be always chosen so as to fix $R$ element-wise (cf. Chapter I in [7]).

From (R.2), any $f(z)$ in $M(R)$ may be considered to be defined on $R^{*}$ as a continuous function on $R^{*}$. From (R.1), this extension is unique. So there occurs no confusion if we use the same letter $f$ for this extended function. Further $\|f\|=\sup _{R}|f(z)|=\sup _{R^{\ddagger}}|f(p)|$ by (R.1). Thus we may consider that $M(R)$ is a subalgebra of $C\left(R^{*}\right)$ which is the totality of continuous functions 
defined on $R^{*}$ with the norm $\|f\|=\sup _{n^{*}}|f(p)|$. From Weierstrass-Stone's approximation theorem (cf. Bourbaki $[1]$ ), $M(R)$ is dense in $C\left(R^{*}\right)$ with respect to the uniform norm.

Now we put $\Delta=\left\{p ; p\right.$ is in $R^{*}$ and $f(p)=0$ for all $f$ in $\left.M_{\Delta}(R)\right\}$. It is clear that the set $\Delta$ is a compact subset of $R^{*}-R$. Following Royden [9] we call $\Delta$ the harmonic boundary of $R$. We set

$$
C_{\Delta}\left(R^{*}\right)=\left\{f(p) ; f \text { belongs to } C\left(R^{*}\right) \text { and vanishes on } \Delta\right\} .
$$

Evidently $M_{\Delta}(R)$ is contained in $C_{\Delta}\left(R^{*}\right)$. The following simple fact plays an important role and has a significant function theoretic meaning which will be clarified later.

Lemma 1.3. The closure of $M_{\dot{4}}\left(R^{R}\right)$ in $C\left(R^{*}\right)$ with respect to the uniform norm coincides with $C_{\Delta}\left(R^{*}\right)$.

Proof. Let $M_{\Delta}(\bar{R})$ be the closure of $M_{\Delta}(R)$ in $C\left(R^{*}\right)$ with respect to the uniform norm. By Lemma 1.2, $M_{\Delta}(R)$ is a closed ideal of $C\left(R^{*}\right)$. Hence it is well known in the theory of function algebra that there exists a compact subset $F$ of $R^{*}$ such that $M_{\triangle}(R)$ is the totality of functions in $C\left(R^{*}\right)$ which vanishes on $F$. From $\bar{M}_{\Delta}(R) \subset C_{\lrcorner}\left(R^{*}\right), F$ must coincides with $\Delta$. This completes the proof.

For later use, we state the following criterion due to Royden [8];

Lemma 1.4. The following statements are mutually equivalent: $(a) R$ is of parabolic type; (b) the constant function 1 belongs to $M_{\Delta}(R)$; (c) the constant function 1 belongs to $C_{\Delta}\left(R^{*}\right)$; (d) 4 is empty.

Proof. As for the equivalence of (a) and (b), we refer to Royden [8]. The equivalence of $(b)$ and $(d)$ is evident. Thus we have only to show the implication (c) $\rightarrow$ (b) since the inverse implication is trivial. By Lemma 1.3, there exists a function $f(z)$ in $M_{\Delta}(R)$ such that $f(z)>1 / 2$ on $R$ since 1 belongs to $C_{\Delta}\left(R^{*}\right)$. As $1 / f(z)$ belongs to $M(R)$ and $M_{\Delta}(R)$ is an ideal of $M(R)$ (Lemma 1.2), so $1=(1 / f(z)) f(z)$ belongs to $M_{\Delta}(R)$.

This completes the proof.

3. Double of normal subdomains. Let $D$ be a subdomain of a Riemann surface $R$. Assume that the relative boundary $\Gamma$ of $D$ with respect to $R$ consists of at most enumerably many analytic Jordan curves $\{\gamma\}$. The curves $\{\gamma\}$ are 
assumed to be closed or open with no end points in $R$. Furthermore $\{r\}$ has no point of accumulation in $R$. In this case we say that $D$ is a normal subdomain of $R$.

Let $D_{1}$ and $D_{2}$ be two copies of $D$. We put $\hat{D}=D_{1} \cup D_{2} \cup r$. We introduce a conformal structure into $\hat{D}$ as follows. We give $D_{1}$ the relative conformal structure of $R$ and extend this structure to $D_{2} \cup \Gamma$ by the symmetrization with respect to $\Gamma$. We say that $\dot{D}$ is the double of $D$ with respect to $\Gamma$. A function $f(z)$ on $D \cup I$ is naturally extended to a function $\hat{f}(\hat{z})$ on $\hat{D}$ by defining $\hat{f}(\hat{z})$ $=f(z)$ if $\hat{z}$ corresponds to $z$ in $D \cup I$.

Let a function $f(z)$ be in $M(R)$ (or $C\left(R^{*}\right)$ ). Restrict $f(z)$ to $D \cup I$ and consider a function $\hat{f}(\hat{z})$ on $\hat{D}$ defined as above. We denote by $\hat{M}(R)$ (or $\left.\hat{C}\left(R^{*}\right)\right)$ the totality of such functions $\hat{f}(\hat{z})$ on $\hat{D}$. Similarly $\hat{M}_{\Delta}(R)$ and $\hat{C}_{\Delta}\left(R^{*}\right)$ are defined. Then from the definition, we get (cf. S. Mori [4])

Lemma 1.5. $\hat{M}_{\Delta}(R) \subset M_{\Delta}(\hat{D})$ and $\hat{C}_{\Delta}\left(R^{*}\right) \subset C_{\Delta}\left(\hat{D}^{*}\right)$.

4. Harmonic decomposition. In this section, we exclude the trivial case that $\Delta$ is empty, i.e. we assume that $R$ is of hyperbolic type.

First we prove the following lemma which plays an important role in our argument of this section:

LeMma 1.6. Suppose that a sequence $\left\{f_{n}(z)\right\}$ of real-valued functions in $M_{0}(R)$ and a real-valued function $f(z)$ satisfying the conditions (M.1) and (M.3) are given. If $\left\{f_{n}(z)\right\}$ converges to $f(z)$ in CD-topology, then $f(z)$ vanishes continuously on 4 .

Proof. We put $g(z)=f(z)^{2}\left(1+f(z)^{2}\right)^{-1}$ and $g_{n}(z)=f_{n}(z)^{2}\left(1+f_{n}(z)^{2}\right)^{-1}$. Clearly $g_{n}(z)$ is in $M_{0}(R)$ and bounded and $g(z)$ belongs to $M(R)$. We shall show that $\left\{g_{n}(z)\right\}$ converges to $g(z)$ in $B D$-topology. It is evident that it converges in $B$-topology. So we have to prove that it converges in $D$-topology. By an easy computation, we get

$$
\begin{aligned}
D\left[g_{n}-g\right] & =\iint_{R}\left[\left(a_{n} \frac{\partial}{\partial x}\left(f-f_{n}\right)+b_{n} c_{n} \frac{\partial f}{\partial x}\right)^{2}\right. \\
& \left.+\left(a_{n} \frac{\partial}{\partial y}\left(f-f_{n}\right)+b_{n} c_{n} \frac{\partial f}{\partial y}\right)^{2}\right] d x d y,
\end{aligned}
$$

where $a_{n}=a_{n}(z), b_{n}=b_{n}(z)$ and $c_{n}=c_{n}(z)$ are bounded functions uniformly for all $n$ and 


$$
c_{n}(z)=\left(f(z)-f_{n}(z)\right)\left[\left(1+f(z)^{2}\right)\left(1+f_{n}(z)^{2}\right)\right]^{1 / 2} .
$$

Hence we get

$$
D\left[g_{i 2}-g\right] \leqq 2 \iint_{E} a_{n}^{2} d\left(f-f_{n}\right) \wedge * d\left(f-f_{n}\right)+2 \iint_{R} b_{n}^{2} c_{n}^{2} d f \wedge^{*} d f .
$$

Let $K$ be an arbitrary compact subset of $R$. Then we have

$$
D\left[g_{n}-g\right] \leqq 2 k^{2} D\left[f-f_{n}\right]+2 k^{2} \sup _{h} c_{n}(z)^{2} D[f]+2 k^{4} \iint_{k i-K} d f \wedge^{*} d f,
$$

where $k=\sup \left(\left\|a_{n}\right\|,\left\|b_{n}\right\|,\left\|c_{n}\right\| ; n=1,2, \ldots\right)$. Thus we get

$$
\lim D\left[g_{n}-g\right] \leqq 2 k^{1} \iint_{R-K} d f \wedge^{*} d f
$$

Letting $K \uparrow R$, we obtain the required property. This shows that $g(z)$ belongs to $M_{\Delta}(R)$ or $g(z)$ vanishes on $\Delta$. Hence $f(z)$ vanishes continuously on $\Delta$.

This completes the proof.

Now we introduce a new class of functions on $R$, i.e. we denote by $F(R)$ the totality of functions on $R$ satisfying (M.1) and (M.3). We also denote by $F^{\prime}(R)$ the totality of positive members in $F(R)$.

Here we remark the following elementary fact. Let $f(x)$ be a real-valued and absolutely continuous function defined in an interval $(a, b)$. Then by the definition of absolute continuity, it is easy to see that $g(x)=\max (f(x), 0)$ is also absolutely continuous and satisfies

$$
\int_{a}^{b}\left|\frac{d}{d x} g(x)\right|^{2} d x \leqq \int_{a}^{b}\left|\frac{d}{d x} f(x)\right|^{2} d x
$$

From this we get

Lemma 1.7. The totality of real-valued members in $F(R)$ forms a vector lattice under the operations $f(z) \vee 0=\max (f(z), 0)$ and $f(z) \wedge 0=\min (f(z), 0)$. Moreover

$$
D[f(z) \vee g(z)], D[f(z) \wedge g(z)] \leqq D[f(z)-g(z)]+D[g(z)] .
$$

Lemma 1.8. Any function $f(z)$ in $F(R)$ is continuous on $R^{*}$ admitting the infinite values.

Proof. We may assume that $f(z)$ is real-valued. If $g(z)$ is in $F^{+}(R)$, then $h(z)=g(z)(1+g(z))^{-1}$ is in $M(R)$ and so continuous on $R^{*}$. Hence $g(z)$ is continuous on $R^{*}$ admitting the infinite value. Thus the same is true for $f(z) \vee 0$ 
and $f(z) \wedge 0$ (cf. Lemma 1.7). Note that if $f(z) \vee 0=\infty($ or $f(z) \wedge 0=-\infty)$ at a point $p$ in $R^{*}$, then $f(z)>0($ or $f(z)<0)$ and $f(z) \wedge 0=0$ (or $f(z) \vee 0=0$ ) on a neighborhood of $p$. This excludes the case $f(z) \vee 0+f(z) \wedge 0=\infty-\infty$. Hence $f(z)=f(z) \vee 0+f(z) \wedge 0$ is continuous on $R^{*}$ admitting the infinite values.

We denote by $F_{\Delta}(R)$ the totality of functions in $F(R)$ which vanish continuously on $\Delta$. Now we prove the following

Theorem 1.1 (Harmonic decomposition). Any junction $f(z)$ in $F(R)$ is decomposed into the following form:

$$
f(z)=u(z)+g(z),
$$

where $u(z)$ is in $H D(R)$ and $g(z)$ is in $F_{\Delta}(R)$.

Proof. By Lemma 1.7, we may assume that $f(z)$ is non-negative. Let $\left\{R_{n}\right\}_{n=0}$ be an exhaustion of $R$, i.e. the closure of $R_{n}$ is contained in $R_{n+1}$ and $R_{n}$ is compact domain in $R$ with the boundary consists of a finite number of analytic closed Jordan curves and $R=\bigcup_{n=0}^{\infty} R_{n}$. Further we assume that $R_{n}$ is a parameter neighborhood of a point $z_{0}$ in $R$. By Harnack's inequality there exists a finite and positive constant number $k$ such that for any $v(z)$ in $H P(R)$, $v\left(z_{0}\right) \leq k \inf _{U} v(z)$, where $U=R_{0}$.

Let $\widetilde{u}_{n}(z)$ be the solution of Dirichlet problem in $R_{n}$ with the boundary value $f(z)$ on $\partial R_{n}$. We put

$$
u_{n}(z)= \begin{cases}\tilde{u}_{n}(z) & \text { on } R_{n} ; \\ f(z) & \text { on } R-R_{n}\end{cases}
$$

and $g_{n}(z)=f(z)-u_{n}(z)$. Note that $u_{n}(z) \geqq 0$ on $R$. By Green's formula and Dirichlet principle, we have

$$
D[f]=D\left[u_{n}\right]+D\left[g_{n}\right]
$$

and if $n>m$

$$
D\left[u_{n}\right] \leqq D\left[u_{m}\right]
$$

Now we define $\widetilde{w}_{n}(z)$ as the solution of Dirichlet problem in $R_{n}-\bar{U}$ with the boundary values 1 on $\partial U$ and 0 on $\partial R_{n}$. We put

$$
w_{n}(z)= \begin{cases}1 & \text { in } U ; \\ \widetilde{w}_{n}(z) & \text { in } R_{n}-U \\ 0 & \text { in } R-R_{n} .\end{cases}
$$

The sequence $\left\{w_{n}(z)\right\}$ converges to a function $w(z)$ on $R$ in $B D$-topology. By 
our assumption that $\Delta$ is not empty, we get

$$
D\left[w_{n}\right] \geqq D[w]>0 .
$$

By Green's formula, we have

$$
\begin{aligned}
\iint_{R} d g_{n} \wedge * d w_{n} & =\int_{\partial(R-U)} g_{n}{ }^{*} d w_{n}=\int_{\partial U} g_{n}{ }^{*} d w_{n} \\
& =\int_{\partial U} f^{*} d w_{n}-\int_{\partial U} u_{n}^{*} d w_{n},
\end{aligned}
$$

since $g_{n}(z)$ vanishes on $\partial R_{n}$. From this we get

$$
\inf _{\partial U} u_{n}(z) \int_{\partial U} * d w_{n} \leqq \sup _{U} f(z) \int_{\partial U} * d w_{n}+\left|\iint_{R} d g_{n} \wedge * d w_{n}\right| .
$$

By Green's formula and Schwarz's inequality, we obtain

and

$$
D\left[w_{n}\right]=\iint_{R} d w_{n} \wedge^{*} d w_{n}=\int_{\partial\left(R_{n}-C^{\prime}\right)} w_{n}^{*} d w_{n}=\int_{\partial U} * d w_{n}
$$

$$
\iint_{R} d g_{n} \wedge * d w_{n} \leqq\left(D\left[g_{n}\right] D\left[w_{n}\right]\right)^{1 / 2} \leqq\left(D[f] D\left[w_{n}\right]\right)^{1 / 2}
$$

From these, we get by putting $a=\sup _{U} f(z)$

$$
\inf _{\partial U} u_{n}(z) D\left[w_{n}\right] \leqq a D\left[w_{n}\right]+\left(D[f] D\left[w_{n}\right]\right)^{1 / 2} .
$$

Hence we have

$$
u_{n}\left(z_{0}\right) \leqq k a+k(D[f] / D[w])^{1 / 2} .
$$

This shows that the sequence $\left\{u_{n}\left(z_{0}\right)\right\}$ is bounded. Thus $\left\{u_{n}(z)\right\}$ forms a normal family and we may assume that $\left\{u_{n}(z)\right\}$ converges to a harmonic function $u(z)$ in $C$-topology. Hence $\left\{g_{n}(z)\right\}$ converges to $g(z)=f(z)-u(z)$ in $C$-topology.

Now we see by Fatou's lemma that

$$
D[u] \leqq \lim _{n} D\left[u_{n}\right] \leqq D[f] .
$$

So we conclude that $u(z)$ belongs to the class $H D(R)$ and we may suppose

$$
\lim _{m} D\left[u-u_{m}\right]=\lim _{m} D\left[g-g_{m}\right]=0 .^{2)}
$$

2) Since $D\left[u_{n}\right] \leqq D[f]$ and $u_{n} \rightarrow u$ in $C$-topology, by choosing a suitable subsequence, we may assume $d u_{n} \rightarrow d u$ weakly. As $D\left[f, u_{m}\right]=D\left[u_{m}\right]$ and $D[f, u]=D\left[u_{n}, u\right](n=1,2, \ldots)$, so $\lim _{m} D\left[u_{m}\right]=D[f, u]=D\left[u_{u}, u\right]$ and hence $\lim _{m} D\left[u_{m}\right]=D[u]$ by making $n \nearrow \infty$. Thus $\lim _{m} D\left[u-u_{m}\right]=0$. 
This shows that $\left\{g_{n}(z)\right\}$ in $M_{0}(R)$ converges to $g(z)$ in $C D$-topology. Thus by Lemma 1.6, $g(z)$ belongs to $F_{\perp}(R)$ and $f(z)=u(z)+g(z)$.

This completes the proof of Theorem 1.1.

Corollary. (a) (Royden [8]). For the decomposition $f(z)=u(z)+g(z)$ in Theorem 1.1, $u(z)$ is in $H B D(R)$ and $g(z)$ is in $M_{\Delta}(R)$ and $\|u\| \leq\|f\|$ if $f(z)$ belongs to $M(R)$.

(b) If $f(z)$ is positive and subharmonic (or superharmonic), then $u(z) \geqq f(z)$ (or $u(z) \leqq f(z))$ on $R$.

(c) If $v(z) \geqq f(z)>0$ (or $f(z) \geqq 0$ and $f(z) \geqq v(z))$ for some superharmonic (or subharmonic) function defined on $R$, then $u(z) \leqq v(z)$ (or $u(z) \geqq v(z)$ ) on $R$.

Remark. It will be proved in section 6 that any $H D$-function defined on $R$ takes its maximum and minimum on $d$. From this, we see at once that the harmonic decomposition asserted in Theorem 1.1 is unique. Futhermore, the unicity of harmonic decomposition implies that any function $f(z)$ in $F_{\Delta}(R)$ is represented as the limit in $C D$-topology of a sequence of functions belonging to $M_{0}(R)$. From this, $F_{\perp}(R)$ is orthogonal to $H D(R)$ in the sense that

$$
\iint_{R} d f \wedge * d u=0
$$

for $f$ in $F_{\Delta}(R)$ and $u$ in $H D(R)$. Hence in our harmonic decomposition $f(z)$ $=u(z)+g(z)$, it holds

$$
D[f]=D[u]+D[g] .
$$

5. A maximum principle for half bounded functions. We use the following notations for real-valued functions defined on $R$ :

$$
\lim _{z \rightarrow y} f(z)=\inf _{(U)} \sup _{U \cap R} f(z)
$$

and

$$
\lim _{z \rightarrow l,} f(z)=\sup _{\{U\}} \inf _{U \cap R} f(z)
$$

where $\{U\}$ is a neighborhood system of a point $p$ in $R^{*}$. Now we prove

THEOREM 1.2 (Maximum principle I). Suppose that $u(z)$ is real-valued harmonic function defined on $R$ such that $u(z)$ is bounded from above (or below) and 


$$
\lim _{z \rightarrow \zeta} u(z) \leqq M \quad\left(\text { or } \lim _{z \rightarrow \zeta} u(z) \geqq m\right)
$$

at any point $\zeta$ in 4. Then it holds

$$
u(z) \leqq M \quad(\text { or } u(z) \geqq m)
$$

on $R$.

Proof. We have only to prove the theorem under the assumption that $u(z)$ is non-negative on $R$. Assume that

$$
\lim _{z \rightarrow \zeta} u(z) \geqq m
$$

at any point $\zeta$ in $\Delta$. We have to show that $u(z) \geqq m$ on $R$. Contrary to the assertion, suppose that there exists a point $z_{0}$ in $R$ such that $u\left(z_{0}\right)$ is smaller than $m$. We choose a real number $t$ such that

$$
u\left(z_{0}\right)<t<m
$$

and the level curve $(z ; u(z)=t)$ has no point $z$ at which $d u(z)=0$. We take $D$ the component of the open set $(z ; u(z)<t)$ which contains the point $z_{0}$. It is evident that $D$ is a normal subdomain of $R$. It is also clear that $v(z)$ $=u(z)-t$ belongs to the class $H B(D)$, i.e.

$$
-t<v(z)<0 \text {. }
$$

We denote the relative boundary of $D$ by $I$. Let $\hat{D}$ be the double of $D$ with respect to $\Gamma$. Then

$$
v_{0}(\hat{z})=\left\{\begin{aligned}
v(z) & \text { on } D_{1} \\
-v(z) & \text { on } D_{2} \cup T
\end{aligned}\right.
$$

belongs to $H B(\hat{D})$ and is non-constant, where $\hat{D}=D_{1} \cup D_{2} \cup T$ and $z$ is the corresponding point in $D$ to $\hat{z}$. Hence, of course, $\hat{D}$ does not belong to the class of parabolic Riemann surfaces.

On the other hand,

$$
\lim _{z \rightarrow \zeta} v(z) \geqq m-t>0
$$

at any point $\zeta$ in $\Delta$. So we can find a neighborhood $U$ of $\Delta$ in $R^{*}$ such that $v(z)>0$ in $U \cap R$. This shows that $D$ is contained in $R^{*}-U$. Thus we can find a continuous function $f(z)$ on $R^{*}$ such that $f(z)$ vanishes on $\Delta$ and 1 on D. Then $f(z)$ belongs to $C_{\Delta}\left(R^{*}\right)$ and $\hat{f}(\hat{z})=1$ on $\hat{D}^{*}$. By Lemma 1.5, $\hat{C}_{\Delta}\left(R^{*}\right)$ is contained in $C_{\Delta}\left(\hat{D}^{*}\right)$, which shows that 1 belongs to $C_{\Delta}\left(\hat{D}^{*}\right)$. Hence by 
Lemma $1.4, \hat{D}$ is of parabolic type. This is absurd since we have seen that $\hat{D}$ is not of parabolic type.

This completes the proof of Theorem 1.2.

This theorem will be extended in Theorem 2.4.

Corollary. (a) Suppose that a real-valued HB-function $u(z)$ defined on $R$ satisfies

$$
m \leqq \lim _{z \rightarrow \zeta} u(z) \leqq \lim _{z \rightarrow \zeta} u(z) \leqq M
$$

at any point $\zeta$ in 4 . Then it holds

$$
m \leqq u(z) \leqq M
$$

at any point on $R$.

(b) (Mori-Ota [5]). Any real-valued HBD-function defined on $R$ takes its maximum and minimum on 4.

6. A maximum principle for harmonic functions with finite Dirichlet integrals. In section 4 , we have proved that $H D$-functions are continuous on $R^{*}$. In this section, we shall prove

Theorem 1.3 (Maximum principle II). Any real-valued HD-function $u(z)$ defined on $R$ takes its maximum and minimum on $\Delta$.

Proof. Let $M=\sup _{R} u(z)$ and $m=\inf _{R} u(z)$. We have only to prove that $u(z)$ attains $M$ on $\Delta$ since the assertion concerning $m$ can be proved if we replace $u(z)$ by $-u(z)$. Moreover we may assume $M=\infty$. For if $M<\infty$, the assertion is contained in Theorem 1.2.

Now consider the function $u(z) \vee 0$ on $R$. Then $u(z) \vee 0$ is in $F^{+}(R)$ and we may apply Theorem 1.1 and (b) in Corollary of Theorem 1.1 to conclude

$$
u(z) \vee 0=v(z)+g(z)
$$

and

$$
v(z) \geqq u(z) \vee 0,
$$

where $v(z)$ belongs to $H D(R)$ and $g(z)$ vanishes continuously on $\Delta$. From this we have

$$
\sup _{R} v(z)=\sup _{R} u(z) \vee 0=\sup _{R} u(z)=\infty
$$

and

$$
\max _{\Delta} v(\zeta)=\max _{\Delta} u(\zeta) \vee 0=\max _{\Delta} u(\zeta)
$$


Hence we have only to prove that $\max _{\Delta} v(\zeta)=\infty$ if $\sup _{R} v(z)=\infty$.

Contrary to the assertion, assume that

$$
c=\sup _{\Delta} v(\zeta)<\infty .
$$

Consider $v(z) \wedge n$ for positive integers $n>c$. This belongs to $M(R)$. By Theorem 1.1 and Corollary (b) of Theorem 1.2, there exists $w(z)$ in $H B D(R)$ such that $\|w\|=c$ and

$$
v(z) \wedge n=w(z)+g_{n}(z) \quad(n>c),
$$

where $g_{n}(z)$ vanishes on $\Delta$ and from the proof of Theorem $1.1 g_{n}(z)$ are limiting functions of functions in $M_{0}(R)$ in $C D$-topology since $v(z) \wedge n \geqq 0$. Hence we have

$$
\iint_{R} d h \wedge * d g_{n}=0
$$

for all $h(z)$ in $H D(R)$. Using this we easily get

$$
D[v(z)-w(z)] \leq D[v(z)-v(z) \wedge n] \searrow 0
$$

as $n \zeta \infty$. Thus $v-w$ is a constant and vanishes on $\Delta$. So $v=w$ and hence $\|v\|=c$, which contradicts to $\sup _{R} v(z)=\infty$.

This completes the proof of Theorem 1.3.

\section{A measure on the harmonic boundary}

1. Canonical measure. To investigate harmonic functions defined on the unit disc $U$, the Poisson integral plays an important role. In this case, the Lebesgue measure $m$ on the unit circumference $C$ is completely determined by the following two properties:

(m.1) $m$ is a regular outer measure which makes compact sets measurable;

(m.2) there exists a kernel function $P(\zeta, z)$ defined on $C \times U$ which is non-negative and $m$-integrable for every fixed $z$ such that

$$
m(X)=\int_{X} P(\zeta, 0) d m(\zeta)
$$

for every $m$-measurable sets $X$ in $C$ and

$$
u(z)=\int_{C} u(\zeta) P(\zeta, z) d m(\zeta)
$$

for every $u(z)$ in $S$ which is an arbitrary fixed subset of $H(\bar{U})$ such that $S$ is 
dense in $H(\bar{U})$ with respect to the uniform norm, where $H(\vec{U})$ denotes the totality of continuous functions defined on $U \cup C$ and harmonic on $U$.

In view of this, we shall define a measure on the harmonic boundary $A$ of a Riemann surface $R$ along with the above direction.

Granting that $R$ is $U$ and $A$ is $C$, we may take $H B D(R)$ as $S$. Thus we are led to the following definition.

Definition of the canonical measure $\mu$ on $\Delta$. Fix a point $O$ in $R$. A measure $\mu$ on $\Delta$ is called the canonical measure on $\Delta$ with the origin at 0 if it satisfies the following two conditions:

( $\mu .1) \mu$ is a regular Borel measure on $\Delta$;

( $\mu .2)$ there exists a kernel function $K(\zeta, z)$ on $\Delta \times R$ which is non-negative and $\mu$-integrable for all fixed $z$ in $R$ such that

$$
\mu(X)=\int_{X} K(\zeta, 0) d \mu(\zeta)
$$

for every Borel sets $X$ in $A$ and

$$
u(z)=\int_{\Delta} u(\zeta) K(\zeta, z) d \mu(\zeta)
$$

for every $u(z)$ in $H B D(R)$. The kernel function $K(\zeta, z)$ is called a harmonic kernel belonging to $\mu$.

First we prove

Theorem 2.1. There exists one and only one canonical measure $\mu$ on $A$ with the origin at 0 .

Proof. Let $H\left(R^{*}\right)$ be the totality of continuous functions defined on $R^{2}$ which are harmonic in $R$. Let $M(\Delta)$ be the totality of restrictions to $\Delta$ of functions in $M(R)$. As $M(R)$ is dense in $C\left(R^{*}\right)$ with respect to the uniform norm, $M(\Delta)$ is dense in $C(\Delta)$ with respect to the uniform norm. Let $f(\zeta)$ be contained in $C(\Delta)$. We can find a sequence $f_{n}(\zeta)$ in $M(\Delta)$ such that $\lim _{n}\left\|f(\zeta)-f_{n}(\zeta)\right\|=0$. Let $\widetilde{f}_{n}(z)$ be an extension of $f_{n}(\zeta)$ to $R^{*}$. Apply the harmonic decomposition to get

$$
\widetilde{f}_{n}(z)=u_{n}(z)+g_{n}(z)
$$

where $u_{n}(z)$ is in $\operatorname{HBD}(R)$ and $g_{n}$ vanishes on 4 . Hence $u_{n}(\zeta)=f_{n}(\zeta)$ and

$$
\left\|u_{n}(z)-u_{m}(z)\right\|=\left\|f_{n}(\zeta)-f_{m}(\zeta)\right\|
$$


by maximum principle. Thus there exists a function $u(z)$ in $H\left(R^{*}\right)$ such that $\lim _{n}\left\|u_{n}-u\right\|=0$. Clearly $u(\zeta)=f(\zeta)$ on $\Delta$. Again by applying maximum principle, we see that such $u(z)$ is unique. Thus we may use the notation $u_{f}(z)$ to denote this $u(z)$.

Now fix an arbitrary point $z$ in $R$. Then $u_{f}(z)$ is a positive linear functional on $C(\Delta)$ : for $f$ and $g$ in $C(\Delta)$ and a complex number $a$,

$$
\begin{aligned}
& u_{a f+g}(z)=a u_{f}(z)+u_{g}(z) ; \\
& u_{f}(z) \geqq 0 \quad \text { if } \quad f(\zeta) \geqq 0 \text { on } \Delta .
\end{aligned}
$$

By Riesz-Markoff-Kakutani's theorem (cf. [3]), there exists a regular Borel measure $\mu_{z}$ such that

$$
u_{f}(z)=\int_{\Delta} f(\zeta) d \mu_{z}(\zeta)
$$

Thus we get a family $\left(\mu_{z} ; z\right.$ is in $R$ ) of regular Borel measures on $\Delta$. Clearly, $\mu_{z}(\Delta)=1$ for all $z$ in $R$.

Let $K$ be a compact subset of $R$. By Harnack's theorem, we can find a positive number $k=k(K)^{3)}$ such that

$$
u\left(z^{\prime}\right) \leqq k u\left(z^{\prime \prime}\right)
$$

for every $u(z)$ in $H P(R)$ and every pairs $\left(z^{\prime}, z^{\prime \prime}\right)$ of points in $K$.

Let $F$ be a compact subset of $\Delta$ and $U$ be an arbitrary open subset of $\Delta$ containing $F$. Define a function $f(\zeta)$ in $C(\Delta)$ as follows:

$$
f(\zeta)= \begin{cases}1 & \text { on } F ; \\ 0 & \text { on } \Delta-U\end{cases}
$$

and $0 \leqq f(\zeta) \leqq 1$ on $\Delta$. For this $f$, we get

As we have

$$
u_{f}\left(z^{\prime}\right) \leqq k u_{f}\left(z^{\prime \prime}\right)
$$

$$
\mu_{z^{\prime}}(K)=\int_{K} f(\zeta) d \mu_{z^{\prime}}(\zeta) \leqq \int_{\Delta} f(\zeta) d \mu_{z^{\prime}}(\zeta)=u_{f}\left(z^{\prime}\right)
$$

and

$$
\mu_{z^{\prime \prime}}(U)=\int_{U^{\prime}} d \mu_{z^{\prime \prime}}(\zeta) \geqq \int_{U} f(\zeta) d \mu_{z^{\prime \prime}}(\zeta)=u_{f}\left(z^{\prime \prime}\right),
$$

so we get

3) For convenience, we shall say that such a possibly small number $k(K)$ as Harnack's constant of $K$. 


$$
\mu_{z^{\prime}}(K) \leqq k \mu_{z^{\prime \prime}}(U)
$$

As $\mu_{z^{\prime \prime}}$ is a regular Borel measure and $U$ is an arbitrary open subset of $\Delta$ containing $K$, so we can conclude that

$$
\mu_{z^{\prime}}(K) \leqq k \mu_{z^{\prime \prime}}(K) .
$$

From the above inequality, we obtain

$$
\mu_{z^{\prime}}(X) \leqq k \mu_{z^{\prime \prime}}(X)
$$

for every Borel set $X$ in $\Delta$.

Now we put $\mu=\mu$. By the Radon-Nikodym theorem, there exists a $\mu$ integrable non-negative function $K(\zeta, z)$ to every $z$ in $R$ such that

$$
d_{\mu z}(\zeta)=K(\zeta, z) d \mu(\zeta) .
$$

It is clear that $\mu$ and $K(\zeta, z)$ thus defined satisfy the conditions $(\mu .1)$ and ( $\mu .2)$. Hence we have shown the existence of canonical measure.

Next we have to prove the unicity of $\mu$. Let $\mu^{\prime}$ be another canonical measure and $K^{\prime}(\zeta, z)$ be the harmonic kernel belonging to $\mu^{\prime}$. We denote

$$
d \mu_{z}^{\prime}=K^{\prime}(\zeta, z) d \mu^{\prime} .
$$

As we have seen above, $H B D(R)$ is dense in $H\left(R^{*}\right)$ with respect to the uniform norm, so we can easily conclude that

$$
u_{f}(z)=\int_{\Delta} f(\zeta) d \mu_{z}^{\prime}(\zeta)
$$

Hence $\mu_{z}^{\prime}=\mu_{z}$ for all $z$ in $R$. In particular, $\mu^{\prime}=\mu^{\prime}=\mu^{\prime}=\mu$.

This completes the proof of Theorem 2.1.

From the proof, we easily get

Corollary. (a) The harmonic kernel belonging to $\mu$ is unique in the following sense. If $K(\zeta, z)$ and $K^{\prime}(\zeta, z)$ are harmonic kernels belonging to $\mu$, then $K(\zeta, z)=K^{\prime}(\zeta, z)$-almost everywhere for each fixed $z$.

(b) Let $\mu_{k}$ be canonical measures on $\Delta$ with the origin at $0_{k}$ in $R(k=1,2)$ and $K_{k}(\zeta, z)$ be their harmonic kernels. Then there exists a system $\left(\varphi_{i j} ; i, j\right.$ $=1,2)$ of non-negative and integrable Borel functions such that $\varphi_{i j}(\zeta) \varphi_{j i}(\zeta)=1$ except a set of measure zero and

$$
d \mu_{i}(\zeta)=\varphi_{i j}(\zeta) d \mu_{j}(\zeta)
$$


and

$$
K_{i}(\zeta, z)=K_{j}(\zeta, z) \varphi_{j i}(\zeta)
$$

except a set of measure zero for each fixed $z$.

Now we fix an origin $O$ in $R$ and consider the canonical measure $\mu$ on $\Delta$ with the origin 0 and the harmonic kernel $K(\zeta, z)$ belonging to $\mu$.

Proposition 2.1. (a) $\mu(\Delta)=1$. (b) If $G$ is a non-empty open subset of $\Delta$, then $\mu(G)>0$. (c) if $f(\zeta)$ belongs to $C(\Delta)$, then

$$
u(z)=\int_{\Delta} f(\zeta) K(\zeta, z) d \mu(\zeta)
$$

is in $H\left(R^{*}\right)$, which is the class of functions continuous on $R^{*}$ and harmonic in $R$. (d) Let $k$ be the Harnack's constant of a compact set $K$ in $R$ and $X$ be an arbitrary Borel set in 4 . Then

$$
\int_{X} K(\zeta, z) d \mu(\zeta) \leqq k \int_{X} K\left(\zeta, z^{\prime}\right) d \mu(\zeta)
$$

for any points $z$ and $z^{\prime}$ in $K$.

Proof. (a), (c) and (d) are clear from the proof of Theorem 2.1. To see (b), we choose a non-empty closed set $F$ contained in $G$. Let $f(\zeta)$ be in $C(\Delta)$ with $0 \leqq f(\zeta) \leqq 1$ on $\Delta$ such that $f(\zeta)=1$ on $F$ and $\theta$ outside $G$. Then $u_{f}(z)$ is non-zero and non-negative. Hence

$$
\mu(G) \geqq \int_{\Delta} f(\zeta) K(\zeta, 0) d \mu(\zeta)=u_{f}(0)>0
$$

Our proof is completed.

2. Elementary properties of integral representation. Let / be the canonical measure on $\Delta$ with the origin 0 and $K(\zeta, z)$ be the harmonic kernel belonging to $\mu$. Now we prove the following fundamental

THEOREM 2.2. Suppose that $f(\zeta)$ is an $\mu$-integrable Borel function on 4. Then the function $u(z)$ defined by

$$
u(z)=\int_{\perp} f(\zeta) K(\zeta, z) d \mu(\zeta)
$$

is a harmonic function defined on $R$. 
Proof. First we assume that $0 \leqq f(\zeta) \leqq 1$. Fix an arbitrary point $z_{0}$ in $R$. Let $U$ be a neighborhood of $z_{0}$ whose closure is compact in $R$. We denote by $k$ the Harnack's constant of the compact set $\bar{U} \cup(\theta)$. By (d) in Proposition 2.1 , we see that

$$
\int_{X} K(\zeta, z) d \mu(\zeta) \leqq k \int_{X} K(\zeta, 0) d \mu(\zeta)=k \mu(X)
$$

for any Borel set $X$ in $\Delta$ and for any point $z$ in $U$. By Lusin's theorem, there exists a compact subset $F_{n}$ of $\Delta$ such that

$$
\mu\left(\Delta-F_{n}\right)<1 / n
$$

and $f(\zeta)$ is a continuous function defined on $F_{n}$. Now by the extention theorem of Tychonoff, there exists a continuous function $f_{n}(\zeta)$ defined on $\Delta$ such that

on $\Delta$ and

$$
0 \leqq f_{n}(\zeta) \leqq 1
$$

$$
f_{n}(\zeta)=f(\zeta)
$$

on $F_{n}$. We put

$$
u_{n}(z)=\int_{\Delta} f_{n}(\zeta) K(\zeta, z) d \mu(\zeta) .
$$

Then $u_{n}(z)$ belongs to the class $H\left(R^{*}\right)$ and $0 \leqq u_{n}(z) \leqq 1$ on $R$.

Let $z$ be an arbitrary point in $U$. Then we get

$$
\begin{aligned}
u(z)-u_{n}(z) & =\int_{\Delta}\left(f(\zeta)-f_{n}(\zeta)\right) K(\zeta, z) d \mu(\zeta) \\
& =\int_{\Delta-F_{n}}\left(f(\zeta)-f_{n}(\zeta)\right) K(\zeta, z) d \mu(\zeta) .
\end{aligned}
$$

As $\left|f(\zeta)-f_{n}(\zeta)\right|$ is not larger than 1 on $\Delta-F_{n}$ and $\int_{\Delta-F_{n}} K(\zeta, z) d \mu(\zeta) \leqq k \mu\left(\Delta-F_{n}\right)$, so we get

$$
\begin{aligned}
\left|u(z)-u_{n}(z)\right| & \leqq \int_{\Delta-F_{n}}\left|f(\zeta)-f_{n}(\zeta)\right| K(\zeta, z) d \mu(\zeta) \\
& \leqq \int_{\Delta-F_{n}} K(\zeta, z) d \mu(\zeta)<k / n .
\end{aligned}
$$

Hence the sequence $\left\{u_{n}(z)\right\}$ of harmonic functions converges to $u(z)$ uniformly on a neighborhood of $z_{0}$. Since $z_{0}$ was arbitrary, $\left\{u_{n}(z)\right\}$ converges to $u(z)$ in $C$-topotogy. Thus $u(z)$ is harmonic on $R$.

Next we assume that $f(\zeta) \geqq 0$. We put $f(\zeta) \wedge n=\min (f(\zeta), n)$. Then by the above, we see that 


$$
v_{n}(z)=\int_{\Delta}(f(\zeta) \wedge n) K(\zeta, z) d \mu(\zeta)
$$

is harmonic on $R$ and

$$
v_{n}(z) \leqq v_{n+1}(z)
$$

at every point in $R$. Furthermore,

$$
\begin{aligned}
u(z) & =\int_{\Delta} f(\zeta) K(\zeta, z) d \mu(\zeta)=\int_{\Delta} \lim _{n}(f(\zeta) \wedge n) K(\zeta, z) d \mu(\zeta) \\
& =\lim _{n} v_{n}(z) .
\end{aligned}
$$

Hence $u(z)$ is harmonic on $R$.

In the general case, $f(\zeta)$ can be represented as a linear combination of positive $\mu$-integrable functions. Hence by reducing to the above, the proof of Theorem 2.2 is over.

Remark. As is easily seen from the above proof, the function $u(z)$ in Theorem 2.2 is represented as a limit of a sequence of $H B D$-functions in $C$ topology.

As for the boundary behaviour of $u(z)$ in Theorem 2.2, we can prove the following (see also Lemma 3.3)

THEOREM 2.3. If $f(\zeta)$ is a bounded Borel function defined on 4 and continuous at a point $\zeta_{0}$ in 4 , then

$$
\lim _{z \rightarrow \zeta_{0}} u(z)=f\left(\zeta_{0}\right),
$$

where

$$
u(z)=\int_{\Delta} f(\zeta) K(\zeta, z) d \mu(\zeta)
$$

Proof. We may assume that $|f(\zeta)| \leqq 1 / 2$ on $\Delta$. Let $\varepsilon$ be an arbitrary positive number. Let $U$ and $V$ be open neighborhoods of $\zeta_{0}$ in $R^{*}$ such that $U$ contains the closure of $V$ in $R^{*}$ and

$$
\left|f(\zeta)-f\left(\zeta_{0}\right)\right|<\varepsilon
$$

for any $\zeta$ in $\Delta \cap U$. We choose a function $g(\zeta)$ in $C(\Delta)$ such that $0 \leqq g(\zeta) \leqq 1$ on $\Delta$ and

$$
g(\zeta)= \begin{cases}0 & \text { on } V \cap \Delta ; \\ 1 & \text { on }\left(R^{+}-U\right) \cap \Delta\end{cases}
$$

As $f\left(\zeta_{0}\right)=\int_{\Delta} f\left(\zeta_{0}\right) K(\zeta, z) d \mu(\zeta)$, so we get 


$$
\begin{aligned}
u(z)-f\left(\zeta_{0}\right) \mid & =\int\left(f(\zeta)-f\left(\zeta_{0}\right)\right) K(\zeta, z) d \mu(\zeta) \mid \\
& \leqq \int_{\Delta \cap \mathcal{C}^{\circ}}\left|f(\zeta)-f\left(\zeta_{0}\right)\right| K(\zeta, z) d \mu(\zeta) \\
& +\int_{\Delta \cap\left(R^{*}-U\right)}\left|f(\zeta)-f\left(\zeta_{0}\right)\right| K(\zeta, z) d \mu(\zeta) .
\end{aligned}
$$

By the definition of $U$ and $g(\zeta)$, we have

$$
\int_{\Delta \cap U}\left|f(\zeta)-f\left(\zeta_{0}\right)\right| K(\zeta, z) d \mu(\zeta) \leqq \int_{\Delta} \varepsilon K(\zeta, z) d \mu(\zeta)=\varepsilon
$$

and

$$
\begin{aligned}
\int_{\Delta \cap\left(R^{*}-C\right)} \mid f(\zeta) & -f\left(\zeta_{0}\right) ! K(\zeta, z) d \mu(\zeta) \leqq \int_{\Delta \cap\left(R^{*}-U\right)} K(\zeta, z) d \mu(\zeta) \\
& \leqq \int_{\Delta} g(\zeta) K(\zeta, z) d \mu(\zeta)
\end{aligned}
$$

As $g(\zeta)$ is continuous on $\Delta$ and vanishes on a neighborhood of $\zeta_{0}$, so the function $v(z)$ defined by

$$
v(z)=\int_{\Delta} g(\zeta) K(\zeta, z) d \mu(\zeta)
$$

is a positive harmonic function on $R$ and vanishes continuously at $\zeta_{0}$. From the inequality

$$
\left|u(z)-f\left(\zeta_{0}\right)\right| \leqq \varepsilon+v(z)
$$

and from $\lim _{z \rightarrow \zeta_{0}} v(z)=0$, we obtain

$$
\lim _{z \rightarrow \zeta_{0}}\left|u(z)-f\left(\zeta_{0}\right)\right| \leqq \varepsilon
$$

As $\varepsilon$ is arbitrary, so we conclude that $\lim _{z \rightarrow \zeta_{0}} u(z)=f\left(\zeta_{0}\right)$.

This completes the proof of Theorem 2.3.

3. A maximum principle (an extension of Section 5 in Chapter 1 ). In Chapter 1 , we have proved that an $H P$-function $u(z)$ defined on $R$ with $\lim _{z \rightarrow \zeta} u(z) \geqq m$ at every point $\zeta$ in $\Delta$ satisfies $u(z) \geqq m$ on $R$. In this section, we shall weaken the assumption on the boundary behaviour at $\Delta$ of $u(z)$ as follows: the same conclusion as above holds if we assume merely that $\lim _{z \rightarrow \xi} u(z) \geqq m$ at every points $\zeta$ in $\Delta$ except a set of $\mu$-measure zero.

Let $D$ be a subdomain of $R$ whose relative boundary consists of at most countably many disjoint Jordan curves which have no point of accumulation in $R$. In this case, we say that $D$ is a Jordan subdomain of $R$. The boundary of 
a Jordan subdomain $D$ relative to $R^{*}$ is decomposed into the following three parts :

$\Gamma=$ the relative boundary of $D$ with respect to $R$;

$\Gamma_{\Delta}=$ the totality of boundary points of $D$ relative to $R^{*}$ which are contained in $\Delta$;

$\Gamma_{0}=$ the totality of boundary points of $D$ relative to $R^{*}$ which are not contained in $\Gamma$ or $\Gamma_{\Delta}$.

First we show that $\Gamma_{0}$ is inessential for bounded harmonic functions.

Lemma 2.1. Suppose that $u(z)$ is a real-valued harmonic function defined on a Jordan subdomain $D$ and bounded from below (or above). If $u(z)$ satisfies

$$
\lim _{D \ni z \rightarrow \zeta} u(z) \geqq m \quad\left(\text { or } \lim _{D \ni z \rightarrow \zeta} u(z) \leqq M\right)^{4}
$$

at every point $\xi$ in $\Gamma \cup \Gamma_{\Delta}$, then $u(z)$ satisfies

on $D$.

$$
u(z) \geqq m \quad(\text { or } u(z) \leqq M)
$$

Proof. We have only to prove the lemma in the case that $u(z)$ is bounded from below and $m=0$. Contrary to the assertion, suppose that there exists a point $z_{0}$ in $D$ such that $u\left(z_{0}\right)<0$. Then we can find a number $\lambda$ such that $u\left(z_{0}\right)<\lambda<0$ and $d u(z)$ does not vanish at any point on the level curve $(z$; $u(z)=\lambda)$. Let $G$ be a component of the open set $(z ; u(z)<\lambda)$ containing $z_{0}$. Then clearly $G$ is a normal subdomain of $R$ and contained in $D$.

Now let $\zeta$ be a point in $\Delta$. If $\zeta$ belongs to $I_{\Delta}$, then by the assumption $\lim _{n \ni z \rightarrow \zeta} u(z) \geqq 0>\lambda$, we can find a neighborhood $U(\zeta)$ of $\zeta$ in $R^{*}$ such that $U(\zeta)$ $\cap G$ is empty. If $\zeta$ does not belong to $\Gamma_{\Delta}$, then we can find a neighborhood $U(\zeta)$ of $\zeta$ in $R^{*}$ such that $U(\zeta) \cap D$ is empty and so $U(\zeta) \cap G$ is empty, since $G$ is contained in $D$. Thus we can find a neighborhood $U$ of $\zeta$ such that $U \cap G$ is empty. Then we can find a continuous function $f(z)$ such that $f$ vanishes on $\Delta$ and 1 on $G$. So $f$ belongs to $C_{\Delta}\left(R^{*}\right)$ and the restriction of $f$ to $D$ is identically 1. By Lemma $1.5, C_{\Delta}\left(\hat{G}^{*}\right)$ contains $\hat{C}_{\Delta}\left(R^{*}\right)$ and so contains $\hat{f}(\hat{z})$ $=1$. Thus by Lemma $1.4, \hat{G}$ is of parabolic type.

\footnotetext{
4) $\lim _{D \exists z \rightarrow \zeta} u(z)=\sup _{\langle U \backslash U \cap D} u(z)$, where $\{U\}$ is a neighborhood system of $\zeta$ in $R^{*}$. Similarly, $\lim _{D \ni z \rightarrow \zeta} u(z)=\inf _{\{U\} \cup \cap D} u(z)$.
} 
On the other hand, $v(z)=\grave{\lambda}-u(z)$ satisfies

$$
0<v(z)<\lambda-\inf _{n} u(z)<\infty
$$

and vanishes on the relative boundary of $G$ with respect to $R$. So $G$ admits a non-constant bounded harmonic function $\hat{v}(\hat{z})$ defined by

$$
\hat{v}(\hat{z})=\left\{\begin{aligned}
v(z) & \text { on } G_{1} ; \\
-v(z) & \text { on } G_{2} \cup \Gamma,
\end{aligned}\right.
$$

where $z$ corresponds to $\hat{z}$. Hence $\hat{G}$ is not of parabolic type. This is absurd.

This completes the proof.

Lemma 2.2. Let $D$ be a Jordan domain of $R$ such that $\mu\left(I_{\Delta}\right)=0$. Then $D$ belongs to the class $S O_{H B}$, that is, there exists no non-constant bounded positive harmonic function defined on $D$ vanishing on $\Gamma$.

Proof. We may assume that the origin $O$ of $\mu$ is in $D$ (cf. (b) in Corollary to Theorem 1.1). Contrary to the assertion, suppose that there exists a nonconstant positive harmonic function $u(z)$ defined on $D$ and vanishing on $I$ : By multiplying a suitable positive constant, we may assume

$$
1=\sup _{\zeta \in \Gamma_{\Delta}} \lim _{D \ni z \rightarrow \zeta} u(z) .
$$

Now $I_{\Delta}=\Delta \cap \bar{D}$, where $\bar{D}$ denotes the closure of $D$ in $R^{*}$. Hence $I_{\Delta}$ is a compact subset of $\Delta$ whose measure is 0 . So we can find a sequence $\left\{U_{n}, V_{n}\right\}_{n=1}^{\infty}$ of open subsets of $\Delta$ such that

$$
U_{n} \supset V_{n} \supset U_{n+1} \text { and } U_{n} \supset \dddot{V}_{n},
$$

where $\bar{V}_{n}$ denotes the closure of $V_{n}$ in $\Delta$, for all $n=1,2, \ldots$ and

$$
\mu\left(U_{n}\right)<1 / n .
$$

We choose a function $f_{n}(\zeta)$ in $C(\Delta)$ such that $0 \leqq f_{n}(\zeta) \leqq 1$ on $\Delta$ and

$$
f_{n}(\zeta)= \begin{cases}1 & \text { on } V_{n} ; \\ 0 & \text { outside } U_{n} \text { in } \Delta .\end{cases}
$$

Define a function $u_{n}(z)$ on $R$ by

$$
u_{n}(z)=\int_{\Delta} f_{n}(\zeta) K(\zeta, z) d_{\ell}(\zeta)
$$

Then $u_{n}(z)$ belongs to $H\left(R^{*}\right)$ and is positive on $R$. Put $w_{n}(z)=u_{n}(z)-u(z)$, 
Then

$$
\lim _{n \exists z \rightarrow \zeta} w_{n}(z) \geqq 1-\lim _{n \exists z \rightarrow \zeta} u(z) \geqq 0
$$

at each point $\zeta$ in $\Gamma_{\Delta}$. For each point $\zeta$ in $\Gamma$,

$$
w_{n}(\zeta)=u_{n}(\zeta)-u(\zeta)>0 .
$$

Hence applying Lemma 2.1 , we conclude that $w_{n}(z) \geqq 0$ on $D$ or

$$
u_{n}(0) \geqq u(0)>0 \text {. }
$$

On the other hand,

$$
u_{n}(0)=\int_{\Delta} f_{n}(\zeta) K(\zeta, 0) d_{\mu}(\zeta) \leqq \int_{U_{n}} K(\zeta, 0) d_{\mu}(\zeta)=\mu\left(U_{n}\right)<1 / n .
$$

Thus we obtain

$$
1 / n>u(0)>0
$$

for all $n=1,2, \ldots$ This is absurd. So $G$ belongs to the class $S O_{H i}$.

This completes the proof.

Now we prove an extension of Theorem 1.2:

TheOREM 2.4 (Maximum principle $\mathrm{I}^{\prime}$ ). Suppose that $u(z)$ is real-valued harmonic function defined on $R$ such that $u(z)$ is bounded from above (or below) and

$$
\lim _{z \rightarrow \zeta} u(z) \leqq M \quad\left(\text { or } \lim _{z \rightarrow \zeta} u(z) \geqq m\right)
$$

at any point $\zeta$ in $\Delta$ except a set of $\mu$-measure zero. Then it holds on $R$

$$
u(z) \leqq M \quad(\text { or } u(z) \geqq m) .
$$

Proof. We have only to prove the theorem in the case that $u(z)>0$ on $R$. Assume that

$$
\lim _{z \rightarrow \zeta} u(z) \geqq m
$$

at any point $\zeta$ in $\Delta$ except a set $X$ of measure zero. Contrary to the assertion, suppose that there exists a point $z_{0}$ in $R$ such that $u\left(z_{0}\right)<m$. We can choose a number $\lambda$ such that $u\left(z_{0}\right)<\lambda<m$ and the level curve $(z ; u(z)=\lambda)$ has no point $z$ with $d u(z)=0$. We take $D$ the component of the open set $(u(z)<\lambda)$ which contains the point $z_{0}$. It is clear that $D$ is a normal subdomain of $R$ and so a Jordan subdomain of $R$. Furthermore

$$
I_{\Delta}=\bar{D} \cap A
$$


is clearly contained in $X$. Hence $" /\left(\Gamma_{\Delta}\right)=0$. Thus by Lemma $2.2, D$ belongs to the class $\mathrm{SO}_{H B}$.

On the other hand, $v(z)=\lambda-u(z)$ satisfies $0<v(z)<\lambda$ on $D$ and vanishes on the relative boundary $I$ of $D$ with respect to $D$. Thus $D$ does not belong to the class $\mathrm{SO}_{H i 3}$. This is a contradiction.

This completes the proof of Theorem 2.4.

As a direct consequence of this theorem, we obtain the following

Corollary. Suppose that a real-valued HB-function $u(z)$ on $R$ satisfies

$$
m \leqq \lim _{z \rightarrow \zeta} u(z) \leqq \lim _{z \rightarrow \zeta} u(z) \leqq M
$$

at any point $\zeta$ in $\Delta$ except a set of 11 -measure zero. Then it holds on $R$

$$
m \leqq u(z) \leqq M .
$$

\section{Some positive minimal harmonic functions}

1. Notations. Functions considered in this chapter are all assumed to be real-valued. We denote the totality of real-valued members in a class $\mathfrak{x}$ of functions by the same notation $\mathfrak{X}$. For example, $M(R)$ denotes the totality of realvalued functions contained in Royden's algebra $M(R)$ associated with $R$. By using this convention, there will occur no confusion.

Let $\mathfrak{X}$ be a class of functions defined on $R$. By $\mathfrak{X}^{+}$we denote the totality of non-negative functions in $\mathfrak{X}$. Suppose that $\mathfrak{X}$ forms a vector space. Then $\mathfrak{X}^{+}$ forms a half-vector space, i.e. $a f(z)+g(z)$ belongs to $\mathfrak{X}^{+}$if $f(z)$ and $g(z)$ are in $\mathfrak{X}^{+}$and $a$ is a non-negative number. By ordering $f \geqq g$ in $\mathfrak{x}$, we always mean that $f(z) \geqq g(z)$ at any point $z$ in $R$. By this ordering, $\mathfrak{X}$ (or $\mathfrak{X}^{+}$) becomes an ordered vector (or half-vector) space.

An element $f(z)$ in $\mathfrak{X}^{+}$satisfying the following property is said to be minimal in $\mathfrak{X}$ or $\mathfrak{X}$-minimal: if $f(z)$ is not identically zero and $f(z) \geqq g(z)$ on $R$ for some $g(z)$ in $\mathfrak{X}^{+}$, then there exists a non-negative number $c$ such that $g(z)$ $=c f(z)$ on $R$.

Let $H(R)$ be the totality of harmonic functions on $R$. Then $H(R)$ is an ordered vector space. For functions $u(z)$ and $v(z)$ in $H(R)$, suppose that there exists a function $w(z)$ in $H(R)$ such that

$$
w(z) \leqq \boldsymbol{u}(z) \text { and } v(z) \quad(\text { or } w(z) \geqq u(z) \text { and } v(z))
$$

and if $w^{\prime}(z)$ in $H(R)$ satisfies the same relation as above, then 


$$
w(z) \geqq w^{\prime}(z) \quad\left(\text { or } w(z) \leqq w^{\prime}(z)\right) .
$$

In this case, we use the following notations:

$$
w(z)=(u \wedge v)(z) \quad(\text { or } w(z)=(u \vee v)(z)) .
$$

As before, we also use the notation $u(z) \wedge v(z)$ (or $u(z) \vee v(z)$ ) to denote the function $\min (\mathrm{u}(z), v(z))$ (or $\max (u(z), v(z))$.

It is easy to see that a necessary and sufficient condition for the existence of $(u \vee v)(z)$ (or $(u \wedge v)(z)$ ) is that there exists a function $w(z)$ in $H(R)$ such that $w(z) \geqq u(z)$ and $v(z)$ (or $w(z) \leqq u(z)$ and $v(z)$ ).

2. Lattice property of $H D(R)$. First we prove

THEOREM 3.1. The class $H D(R)$ (or $H B D(R))$ is a vector lattice under the operations $u \vee v$ and $u \wedge v$.

Proof. We shall only prove the theorem for $H D(R)$. The proof for $H B D(R)$ is quite similar to that for $H D(R)$.

First we prove that $u \vee v$ exists and belongs to $H D(R)$ if $u(z)$ and $v(z)$ belong to $H D(R)$. We may assume that $v(z)=0$. In fact, if $u^{\prime} \vee 0$ exists and belongs to $H D(R)$ for any $u^{\prime}$ in $H D(R)$, then $(u-v) \vee 0+v$ is nothing but $u \vee v$ and belongs to $H D(R)$.

Apply the harmonic decomposition to $u(z) \vee 0$. Then we get $u(z) \vee 0$ $=w(z)+g(z)$, where $w$ is in $H D(R)$ and $g(z)$ vanishes continuously on $\Delta$. By (b) in corollary of Theorem $1.1, w(z) \geqq u(z) \vee 0 \geqq u(z)$ and 0 . If $w^{\prime}(z)$ satisfies $w^{\prime}(z) \geqq u(z)$ and 0 , then $w^{\prime}(z) \geqq u(z) \vee 0$. By (c) in Corollary of Theorem 1.1. we get $w^{\prime}(z) \geqq w(z)$. Hence $w(z)=(u \vee v)(z)$ and belongs to $H D(R)$.

As for the existence of $u \wedge v$, we note that $-(-u) \vee(-v)$ is nothing but $u \wedge v$. Hence we have proved that $H D(R)$ is a vector lattice under $u \vee v$ and $u \wedge v$.

Corollary 1. Suppose that $u(z)$ and $v(z)$ belong to $H D(R)$. Then

$$
u(z) \vee v(z)=(u \vee v)(z)+g(z)
$$

and

$$
u(z) \wedge v(z)=(u \wedge v)(z)+g^{\prime}(z),
$$

where $g(z)$ and $g^{\prime}(z)$ vanish continuously on $\Delta$. In other word,

$$
(u \vee v)(z)=\int_{4}(u(\zeta) \vee v(\zeta)) K(\zeta, z) d_{l \iota}(\zeta)
$$


and

$$
(u \wedge v)(z)=\int_{\Delta}(u(\zeta) \wedge v(\zeta)) K(\zeta, z) d \ell(\zeta)
$$

Proof. By the proof of Theorem 3.1, we have

$$
(u(z)-v(z)) \vee 0=((u(z)-v(z)) \vee 0)(z)+g(z),
$$

where $g(z)$ vanishes continuously on $\Delta$. On the other hand,

$$
(u(z)-v(z)) \vee 0=(u(z)-v(z)) \vee 0+v(z)
$$

and

$$
(u \vee v)(z)=((u-v) \vee 0)(z)+v(z)
$$

Hence we get the first identity. Similarly, the validity of the second identity can be shown.

As for the integral representation, we have only to show that a function $u(z)$ in $H D^{+}(R)$ can be represented as

$$
u(z)=\int_{\Delta} u(\zeta) K(\zeta, z) d \mu(\zeta)
$$

In fact, as $\{(u \wedge n)(z)\}$ is a non-decreasing sequence, so there exists a harmonic function $v(z)$ such that $v(z)=\lim (u \wedge n)(z)$. From

$$
D[(u \wedge n)(z)] \leqq D[u(z) \wedge n] \leqq D[u]
$$

and $(u \wedge n)(z) \leqq u(z)$ on $R$, we get $D[v] \leqq D[u]$ and $u(\zeta) \wedge n \leqq v(\zeta) \leqq u(\zeta)$ on $\Delta$ for all $n$ and so $u(\zeta)-v(\zeta)$ vanishes on the harmonic boundary. Hence by maximum principle II, we get $u(z)=v(z)$ on $R$.

Assume that $u(z)$ is non-negative. Then we get

$$
\begin{aligned}
u(z) & =\lim (u \wedge n)(z)=\lim _{n} \int_{\Delta}(u(\zeta) \wedge n) K(\zeta, z) d \mu(\zeta) \\
& =\int_{\Delta}\left(\lim _{n}(u(\zeta) \wedge n) K(\zeta, z) d \mu(\zeta)\right. \\
& =\int_{\Delta} u(\zeta) K(\zeta, z) d_{\mu}(\zeta) .
\end{aligned}
$$

In the general case, apply the Jordan decomposition to $u(z)$. Then we get the required identity. This completes the proof.

Corollary 2 (Precise form of Virtanen's theorem). For any $u(z)$ in $H D(R)$, there exists a sequence $\left\{u_{n}\right\}$ in $H B D(R)$ converging to $u(z)$ in CD-topology. 
Proof. First we assume that $u(z) \geqq 0$. By the proof of Corollary 2, we see that $(u \wedge n)(z)$ in $H B D$ converges to $u(z)$ in $C$-topology and by using $u(z)$ $+n=(u \vee n)(z)+(u \wedge n)(z)$, we get $D[u(z)-(u \wedge n)(z)]=D[(u \vee n)(z)]$ $\leqq D[u(z) \vee n] \rightarrow 0$ as $n \rightarrow \infty$.

In the general case, let $u(z)=v(z)-w(z)$ be the Jordan decomposition in HD. Then $u_{n}(z)=(v \wedge n)(z)-(w \wedge n)(z)(n=1,2, \ldots)$ satisfy our assertion. This completes the proof.

Let $F(\Delta)$ and $M(\Delta)$ be the totality of restrictions on $\Delta$ of functions in $F(R)$ and $M(R)$ respectively. We obtain

COROLlaRy 3. The classes $F(\Delta)$ and $M(\Delta)$ are vector lattices under the operations $\max (f, g)$ and $\min (f, g)$ and are isomorphic to $H D(R)$ and $H B D(R)$ respectively as vector lattices.

As an application of this, we get

Lemma 3.1 (S. Mori [4]). Suppose that 4 is decomposed into the disjoint open and closed subsets $\Delta_{1}, \ldots, \Delta_{n}$ and a system $\left(a_{1}, \ldots, a_{n}\right)$ of real numbers $a_{k}$ is given. Then there exists a function $f(\zeta)$ in $M(\Delta)$ such that $f(\zeta)=a_{k}$ on $\Delta_{k}$.

Proof. We have only to prove the lemma in the case $\left(a_{1}, \ldots, a_{n}\right)=(1,0$, $\ldots, 0)$. Put $g(\zeta)=2$ on $\Delta_{1}$ and -1 on $\Delta-\Delta_{1}$. Then $g(z)$ is in $C(\Delta)$. As $M(\Delta)$ is dense in $C(\Delta)$ with respect to the uniform norm, we can find a function $h(\zeta)$ in $M(\Delta)$ such that $h(\zeta)$ is arbitrarily close to $g(\zeta)$. Hence we may assume that $h(\zeta)>1$ on $\Delta_{1}$ and $h(\zeta)<0$ on $\Delta-\Delta_{1}$. Then

$$
f(\zeta)=(h(\zeta) \vee 0) \wedge 1
$$

is the required function. This completes the proof.

3. HD-minimal functions. If $\Delta$ contains an isolated point $\zeta_{0}$, then by Lemma 3.1 , we can find in $M(\Delta)$ a function $\%\left(\zeta ; \zeta_{0}\right)$ which equals 1 at $\zeta_{0}$ and vanishes elsewhere on $\Delta$. We shall denote by $w\left(z ; \zeta_{0}\right)$ the function $H B D(R)$ whose boundary value on $\Delta$ is $\chi\left(\zeta ; \zeta_{0}\right)$. We may call $w\left(z ; \zeta_{0}\right)$ as the harmonic measure of $\zeta_{0}$.

Now we state the following topological characterization of $H D$-minimality:

THEOREM 3.2. If $v(z)$ is HD-minimal, then there exists an isolated point $5_{0}$ in $\Delta$ and a positive number $c$ such that

$$
v(z)=c w\left(z ; \zeta_{0}\right) .
$$


Conversely, if $\zeta_{0}$ is an isolated point in 4 , then $w\left(z ; \zeta_{n}\right)$ is HD-minimal.

Proof. Suppose that $v(z)$ is $H D$-minimal. We denote by $v(\zeta)$ the boundary function of $v(z)$ on $\Delta$. Suppose that there exist two points $\zeta_{1}$ and $\zeta_{2}$ in $\Delta$ such that $v\left(\zeta_{k}\right)>0(k=1,2)$. Let $U$ be a neighborhood of $\zeta_{1}$ such that $U$ does not contain $\zeta_{2}$ and $v(\zeta)>c>0$ on $U$. As $M(\Delta)$ is dense in $C(\Delta)$ and is a vector lattice, so we can find a function $g(\zeta)$ in $M(\Delta)$ such that $c \geqq g(\zeta) \geqq 0$ on $\Delta$ and $g\left(\zeta_{1}\right)>0$ and vanishes outside $U$. Then $v(\zeta) \geq g(\zeta)$ on $\Delta$ and by the minimality of $v(z)$ in $H D$ and by the maximum principle II, the $H B D$-function $u(z)$ whose boundary value $g(\zeta)$ must be represented as

$$
u(z)=\eta v(z)
$$

for some positive number $\eta$. Hence $u\left(\zeta_{2}\right)=\eta v\left(\zeta_{2}\right)=0$, which is absurd. Hence $v(z)$ vanishes on $\Delta$ except a point $\zeta_{0}$. Since $v(\zeta)$ is continuous on $\Delta, \zeta_{0}$ is necessarily isolated. Moreover, $\lim (v \wedge n)(z)=v(z)$ in $C$-topology, $(v \wedge n)(z) \neq 0$ for some $n$ and $v(z) \geqq(v \wedge n)(z)$. Hence there exists a positive number $d$ such that $(v \wedge n)(z)=d v(z)$. Thus $v\left(\zeta_{0}\right)$ is finite and $v(\zeta)=v\left(\zeta_{0}\right) w\left(z ; \zeta_{0}\right)$.

Conversely, suppose that $\zeta_{0}$ is an isolated point in $\Delta$ and $w\left(z ; \zeta_{0}\right) \geqq u(z)$ for some $u(z)$ in $H D^{+}(R)$. Then $w\left(\zeta ; \zeta_{0}\right) \geqq u(\zeta) \geqq 0$ shows that $u(\zeta)$ vanishes on $\Delta$ except possibly $\zeta_{0}$. Hence $u(\zeta)=u\left(\zeta_{0}\right) w\left(\zeta ; \zeta_{0}\right)$ and by the maximum principle, $u(z)=u\left(\zeta_{0}\right) w\left(z ; \zeta_{0}\right)$ on $R$. Thus $w\left(z ; \zeta_{0}\right)$ is HD-minimal.

This completes the proof of Theorem 3.2.

Remark. The totality $H\left(R^{*}\right)$ of continuous functions on $R^{*}$ which are harmonic on $R$ coincides with the closure of $H B D(R)$ in $C\left(R^{*}\right)$ with respect to the uniform norm. It is easy to see that $H\left(R^{*}\right)$-minimality is characterized by the isolatedness of a point in 4 . By the above proof, it is clear that $H D$-minimality coincides with $H B D$-minimality.

Hence the concepts " $H D$-minimal", " $H B D$-minimal" and " $H\left(R^{*}\right)$-minimal" coincide with each other.

4. The class $H D(R)$. Constantinescu and Cornea [2] introduced the class $H D(R)$ as follows: a function $u(z)$ defined on $R$ belongs to $H D(R)$ ( or $H B D(R)$ ) if there exists a decreasing sequence $\left\{u_{n}(z)\right\}$ of functions in $H D^{+}(R)$ (or $\left.H B D^{+}(R)\right)$ such that $u(z)=\lim _{n} u_{n}(z)$ at every points $z$ in $R$. Clearly $H D(R)$ (or $H B D(R)$ ) forms an ordered half-vector space and $H B D(R)$ is contained in $H D(R)$. 
Suppose that there exists a decreasing sequence $\left\{u_{n}(z)\right\}$ of non-negative functions in $H\left(R^{*}\right)$. Then there exists a non-negative harmonic function $u(z)$ defined on $R$ such that $u(z)=\lim u_{n}(z)$ at every points $z$ in $R$. In this case, we can conclude that $u(z)$ belongs to $H B D(R)$. In fact, as $H B D(R)$ is dense in $H\left(R^{*}\right)$ with respect to the uniform norm, so we can choose a sequence $\left\{v_{n}(z)\right\}$ in $H B D(R)$ such that $\left\|u_{n}(z)-v_{n}(z)\right\|<1 / n$. Let $w_{n}(z)=v_{n}(z)+1 / n$. Then $w_{n}(z)$ is a decreasing sequence of functions in $H_{B D}(R)$ and $\left\|u_{n}(z)-w_{n}(z)\right\|$ $<2 / n$. Hence $u(z)=\lim w_{n}(z)$ on $R$ and $u(z)$ belongs to the class $H B D(R)$. Thus we may write $H B D(R)=H\left(R^{*}\right)$.

Now let $f(\zeta)$ be a function defined on $\Delta$. Suppose that there exists a decreasing sequence $\left\{f_{n}(\zeta)\right\}$ of functions in $C(\Delta)$ such that $f(\zeta)=\lim _{n} f_{n}(\zeta)$ on $\Delta$. In this case, we say that $f(\zeta)$ is bounded upper semi-continuous on $\Delta$. We denote by $U(\Delta)$ the totality of non-negative bounded upper semi-continuous functions defined on $\Delta$.

First we prove

Lemma 3.2. A function $u(z)$ defined on $R$ belongs to $H B D(R)$ if and only if there exists a function $f(\zeta)$ in $U(\Delta)$ such that

$$
u(z)=\int_{\Delta} f(\zeta) K(\zeta, z) d \mu(\zeta)
$$

Proof. Suppose that $u(z)$ belongs to $H B D(R)$. Then there exists a decreasing sequence $\left\{u_{n}(z)\right\}$ of functions in $H B D^{+}(R)$ such that $u(z)=\lim _{n} u_{n}(z)$. In particular, $\left\{u_{n}(\zeta)\right\}$ is a decreasing sequence of non-negative functions in $C(\Delta)$. Hence we can find a function $f(\zeta)$ in $U(\Delta)$ such that $f(\zeta)=\lim _{n} u_{n}(\zeta)$ on $\Delta$. As

$$
u_{n}(z)=\int_{\Delta} u_{n}(\zeta) K(\zeta, z) d \mu(\zeta)
$$

so we get

$$
\begin{aligned}
u(z) & =\lim \int_{\Delta} u_{n}(\zeta) K(\zeta, z) d_{\mu}(\zeta)=\int_{\Delta} \lim u_{n}(\zeta) K(\zeta, z) d_{\mu}(\zeta) \\
& =\int_{\Delta} f(\zeta) K(\zeta, z) d_{\mu}(\zeta)
\end{aligned}
$$

Conversely, if $u(z)=\int_{\Delta} f(\zeta) K(\zeta, z) d \mu(\zeta)$ for some $f(\zeta)$ in $U(\Delta)$, then there exists a decreasing sequence $\left\{f_{n}(\zeta)\right\}$ of non-negative functions in $C(\Delta)$ such that $f(\zeta)=\lim _{n} f_{n}(\zeta)$. We put 


$$
u_{n}(z)=\int_{\Delta} f_{n}(\zeta) K(\zeta, z) d \mu(\zeta)
$$

Then $u_{n}(z)$ belongs to $H\left(R^{*}\right)$ and $\left\{u_{n}(z)\right\}$ forms a decreasing sequence and

$$
\begin{aligned}
\lim _{n} u_{n}(z) & =\lim _{n} \int_{\Delta} f_{n}(\zeta) K(\zeta, z) d \mu(\zeta) \\
& =\int_{\Delta} \lim _{n} f_{n}(\zeta) K(\zeta, z) d \mu(\zeta)=u(z) .
\end{aligned}
$$

Thus $u(z)$ belongs to $H B D(R)$. This completes the proof.

Lemma 3.3. If $u(z)=\int_{\Delta} f(\zeta) K(\zeta, z) d \mu(\zeta)$ for some $f(\zeta)$ in $U(\Delta)$, then it holds that

$$
\lim _{z \rightarrow \zeta} u(z)=f(\zeta)
$$

at every points $\zeta$ in $\Delta$ except a set of measure zero. Hence the function $f(\zeta)$ uniquely determines (and is determined by) $u(z)$ in $H B D(R)$ except a set of measure zero.

Proof. We can find a decreasing sequence $\left\{f_{n}(\zeta)\right\}$ of non-negative functions in $C(\Delta)$ such that $\lim _{n} f_{n}(\zeta)=f(\zeta)$. Put

$$
u_{n}(z)=\int_{\Delta} f_{n}(\zeta) K(\zeta, z) d_{\mu}(\zeta)
$$

Then $\left\{u_{n}(z)\right\}$ forms a decreasing sequence of non-negative functions in $H\left(R^{*}\right)$ and $u_{n}(z) \geqq u(z)$ and $u(z)=\lim _{n} u_{n}(z)$. If we put $\bar{u}(\zeta)=\lim _{z \rightarrow \zeta} u(z)$ on $\Delta$, then we get

$$
u(\zeta) \leqq u_{n}(\zeta)=f_{n}(\zeta)
$$

on $\Delta$. Hence we have

$$
u(\zeta) \leqq f(\zeta)
$$

Contrary to the assertion, suppose that $\bar{u}(\zeta)<f(\zeta)$ on a subset of measure positive. Then we can find a compact subset $K$ of $\Delta$ with $\mu(K)>0$ and a positive number $\varepsilon$ such that

$$
\vec{u}(\zeta)+\varepsilon<f(\zeta)
$$

on $K$. We set

$$
v(z)=\int_{K} \varepsilon K(\zeta, z) d \mu(\zeta)
$$

Then by Theorem 2.2, $v(z)$ is harmonic on $R$. Moreover $v(z)$ is non-constant 
and $0<v(z) \leqq \varepsilon$ on $R$, since $\mu(K)>0$. By Theorem 2.3 , we see that $\lim _{z \rightarrow \zeta} v(z)=0$ at each point $\zeta$ in $\Delta-K$. Needless to say, $\lim _{z \rightarrow \zeta} v(z) \leqq \varepsilon$ at $\zeta$ in $K$. Hence we have that

$$
\lim _{z \rightarrow \zeta}(u(z)+v(z)) \leqq \bar{u}(\zeta)+\lim _{z \rightarrow \zeta} v(z)
$$

is not larger than $\bar{u}(\zeta)$ on $\Delta-K$ and $\bar{u}(\zeta)+\varepsilon$ on $K$. Thus we have

$$
\lim _{z \rightarrow \zeta}(u(z)+v(z)) \leqq f(\zeta) \leqq u_{n}(\zeta)
$$

at every point $\zeta$ in $\Delta$. From this, we get

$$
\lim _{z \rightarrow \zeta}\left(u_{n}(z)-(u(z)+v(z)) \geqq u_{n}(\zeta)-\lim _{z \rightarrow \zeta}(u(z)+v(z)) \geqq 0\right.
$$

in $\Delta$. Hence by the maximum principle I, we see that

$$
u_{n}(z) \geqq u(z)+v(z) .
$$

making $n \rightarrow \infty$, we finally obtain

$$
u(z) \geqq u(z)+v(z) \geqq 0
$$

on $R$. Thus we see $v(z)=0$ on $R$, which is a contradiction.

This completes the proof.

Summing up these we state

THEOREM 3.3. There exists an order preserving half-vector isomorphism between $H B D(R)$ and $U(\Delta, \iota)$ and this correspondence $u \leftrightarrow f$ is given by

$$
u(z)=\int_{\Delta} f(\zeta) K(\zeta, z) d \mu(\zeta)
$$

where $U(\Delta, \mu)$ denotes the totality of positive bounded upper semi-continuous functions on $\Delta$ in which two functions identical except a set of measure zero are assumed to be the same. Furthermore $\lim _{z \rightarrow \zeta} u(z)=f(\zeta)$ on $\Delta$ except a set of measure zero.

Remark. If we set $V(\Delta)$ be the totality of functions $f$ on $\Delta$ such that there exists a sequence $\left\{f_{n}\right\}$ of functions in $F^{+}(\Delta)$ such that $f_{n} \searrow f$. Identifying two elements in $V(\Delta)$ which coincide on $\Delta$ except a set of measure zero, we get a new class $V(\Delta, \mu)$. Then we get

(a) A function $u$ belongs to $H D(R)$ if and only if there exists a function $f$ in $V(\Delta)$ such that $u(z)=\int_{\Delta} f(\zeta) K(\zeta, z) d \mu(\zeta)$. 
(b) If $u(z)=\int_{\Delta} f(\zeta) K(\zeta, z) d \mu(\zeta)$ for some $f(\zeta)$ in $V(\Delta)$, then $\lim _{z \rightarrow \zeta} u(z)$ $=f(\zeta)$ on $\Delta$ except a set of measure zero. Hence $f$ uniquely determines (and is. determined by) $u$ in $H D(R)$ except a set of measure zero.

By an obvious modification, the proofs of Lemma 3.2 and 3.3 prove the validity of (a) and (b) respectively. Hence we may state

(c) There exists an order preserving half-vector isomorphism between $H D(R)$ and $V(\Delta, \mu)$ and this correspondence $u \leftrightarrow f$ is given by

$$
u(z)=\int_{\Delta} f(\zeta) K(\zeta, z) d_{\mu}(\zeta)
$$

Furthermore $\lim _{z \rightarrow \zeta} u(z)=f(\zeta)$ on $\Delta$ except a set of measure zero.

Corresponding to Theorem 3.1, we prove

THEOREM 3.1'. The class $H D(R)$ (or $H B D(R))$ is a vector lattice under the operations $u \vee v$ and $u \wedge v$.

Proof. We shall only prove the theorem for $H D(R)$. The proof for $H B D(R)$ is same to that for $H D(R)$. It is clear that $(u \wedge v)(z)$ exists and belongs to $H D(R)$ for any $u$ and $v$ in $H D(R)$. So we have only to prove that $(u \vee v)(z)$ exists and belongs to $H D(R)$.

By (a) and (b) in the above remark,

$$
w(z)=\int_{\Delta}(\bar{u}(\zeta) \vee \bar{v}(\zeta)) K(\zeta, z) d \mu(\zeta)
$$

belongs to $H D(R)$, where $\bar{u}(\zeta)=\lim _{z \rightarrow \zeta} u(z)$ and $\ddot{v}(\zeta)=\lim _{z \rightarrow \zeta} v(z)$. Clearly

$$
w(z) \geqq u(z), v(z)
$$

on $R$.

Next suppose that there exists a function $w^{\prime}$ in $H(R)$ such that

$$
w^{\prime}(z) \geqq u(z), v(z)
$$

on $R$. We now prove that $w^{\prime} \geqq w$ on $R$. If we can show

$$
w^{\prime}(z) \geqq \int_{\Delta}((\bar{u}(\zeta) \vee \bar{v}(\zeta)) \wedge n) K(\zeta, z) d \mu(\zeta)
$$

on $R$, then making $n \nearrow \infty$ we get $w^{\prime} \geqq w$. Thus we may assume that

$$
0 \leqq \bar{u}(\zeta), \bar{v}(\zeta) \leqq k<\infty .
$$


By Lusin's theorem we can find a compact set $K_{n}$ in $\Delta$ with the following properties :

$$
\mu\left(\Delta-K_{n}\right)<1 / n
$$

there exists a system of continuous functions $f_{n}$ and $g_{n}$ on $\Delta$ such that

$$
f_{n}=u
$$

and

on $K_{n}$ and

$$
g_{n}=v
$$

$$
0 \leqq f_{n}, g_{n} \leqq k
$$

on $\Delta$. Let $u_{n}, v_{n}$ and $w_{n}$ be functions in $H\left(R^{*}\right)$ with $u_{n}(\zeta)=f_{n}(\zeta), v_{n}(\zeta)=g_{n}(\zeta)$ and $w_{n}(\zeta)=f_{n}(\zeta) \vee g_{n}(\zeta)$ on $\Delta$ respectively. Set

$$
w_{n}^{\prime}(z)=w^{\prime}(z)+\int_{\Delta-K_{n}}\left(f_{n}(\zeta)+g_{n}(\zeta)\right) K(\zeta, z) d \mu(\zeta)
$$

By these definitions, it is easily seen that

$$
w_{n}^{\prime}(z) \geqq u_{n}(z), v_{n}(z)
$$

on $R$. As $u_{n}$ and $v_{n}$ are continuous on $R^{*}$, so we get from the above

$$
\lim _{z \rightarrow \zeta} w_{n}^{\prime}(z) \geqq u_{n}(\zeta), v_{n}(\zeta)
$$

at any point $\zeta$ in $\Delta$. Hence

$$
\lim _{z \rightarrow \zeta} w_{n}^{\prime}(z) \geqq u_{n}(\zeta) \vee v_{n}(\zeta)=w_{n}(\zeta)
$$

or by using the continuity of $w_{n}(z)$ on $R^{*}$

$$
\lim _{z \rightarrow \zeta}\left(w_{n}^{\prime}(z)-w_{n}(z)\right) \geqq 0
$$

at any point $\zeta$ in $\Delta$. Hence by Theorem $1.2, w_{n}(z) \leqq w_{n}^{\prime}(z)$ on $R$ or

$$
w^{\prime}(z)+2 k \int_{\perp-K_{n}} K(\zeta, z) d \mu(\zeta) \geqq \int_{K_{n}}(\bar{u}(\zeta) \vee \bar{v}(\zeta)) K(\zeta, z) d \mu(\zeta) .
$$

Hence by making $n \nearrow \infty, w^{\prime}(z) \geqq w(z)$ on $R$. This completes the proof.

As a direct consequence of Theorem 3.1 , we get the followings.

Corollary 1. Let $u$ and $v$ be in $H D(R)$ and $\bar{u}(\zeta)=\lim _{z \rightarrow \zeta} u(z)$ and $\bar{v}(\zeta)$ $=\varlimsup_{z \rightarrow \zeta} v(z)$ on 4 . Then

$$
u(z)=\int_{\Delta} \bar{u}(\zeta) K(\zeta, z) d \mu(\zeta)
$$


and

$$
v(z)=\int_{\Delta} \bar{v}(\zeta) K(\zeta, z) d \mu(\zeta)
$$

and

$$
(u \vee v)(z)=\int_{\Delta}(\bar{u}(\zeta) \vee \ddot{v}(\zeta)) K(\zeta, z) d \mu(\zeta)
$$

and

$$
(u \wedge v)(z)=\int_{\Delta}(\bar{u}(\zeta) \wedge \tilde{v}(\zeta)) K(\zeta, z) d_{\mu}(\zeta)
$$

Corollary 2. If $u(z)$ belongs to $H D(R)$, then $(u \wedge n)(z)$ belongs to $H B D(R)$ and $u(z)=\lim _{n}(u \wedge n)(z)$ on $R$ in C-topology.

5. Harmouic measure of Borel set in 4 . Let $X$ be a Borel set in $\Delta$. We define a function $w(z ; X)$ by

$$
w(z ; X)=\int_{X} K(\zeta, z) d \mu(\zeta) .
$$

By Theorem $2.1, w(z ; X)$ is a harmonic function defined on $R$ and

$$
0 \leqq w(z ; X) \leqq 1
$$

on $R$. We call $w(z ; X)$ as the harmonic measure of $X$. It is clear that

$$
w(z ; X)=0(\text { or } 1) \text { if and only if } \mu(X)=0 \text { (or } 1) \text {. }
$$

First we begin with the following

Lemma 3.4. Let $K$ be a compact subset of 4 . Then $w(z ; K)$ belongs to the class $H B D(R)$ and $\lim _{z \rightarrow \zeta} w(z ; K)=0$ at each point $\zeta$ in $\Delta-K$ and $\lim _{z \rightarrow \zeta} w(z ; K)=1$ at each point $\zeta$ in $\stackrel{z \rightarrow \zeta}{K}$ except a set of measure zero.

Proof. The first part of our Lemma is a direct consequence of Theorem 2.3. To prove the second part of our lemma, choose a sequence $\left\{U_{n}\right\}$ of open subsets of $\Delta$ such that $U_{n}$ contains the closure of $U_{n+1}$ and $K$ and $\mu\left(U_{n}-K\right)$ $<1 / n$. Suppose that $f_{n}(\zeta)$ is in $C(\Delta)$ and $0 \leqq f_{n}(\zeta) \leqq 1$ on $\Delta$ and $f_{n}(\zeta)=1$ on $\bar{U}_{n+1}$ and 0 outside $U_{n}$ in $\Delta$. Setting $u_{n}(z)=\int_{\Delta} f_{n}(\zeta) K(\zeta, z) d_{\mu}(\zeta)$ and $f(\zeta)$ $=\operatorname{lin}_{n} f_{n}(\zeta)$ on $\Delta$, we see that $\left\{u_{n}(z)\right\}$ and $\left\{f_{n}(\zeta)\right\}$ are decreasing sequences of functions. Hence $f(\zeta)$ belongs to $U(\Delta)$ and

$$
0 \leqq u_{n}(z)-w(z ; K) \leqq \int_{U_{n}-K} K(\zeta, z) d \mu(\zeta)<k / n,
$$


where $k$ is the Harnack's constant of the set $\{0, z\}$. Thus we get

$$
w(z ; K)=\int_{\Delta} f(\zeta) K(\zeta, z) d \mu(\zeta)
$$

which shows that $u(z)$ belongs to $H B D(R)$. By Theorem 3.3,

$$
\lim _{z \rightarrow \zeta} w(z ; K)=f(\zeta)
$$

on $\Delta$ except a set of measure zero. As $f(\zeta)=1$ on $K$, so the proof is completed.

Theorem 3.4. Let $X$ be an arbitrary Borel subset in 4 . Then

$$
\lim _{z \rightarrow \zeta} w(z ; X)=1 \quad \text { a.e. on } X^{5}
$$

and

$$
\lim _{z \rightarrow \zeta} w(z ; X)=0 \quad \text { a.e. on } \Delta-X \text {. }
$$

Proof. Assume that $\mu\left(\zeta ; \zeta\right.$ is in $X$ and $\left.\lim _{z \rightarrow \zeta} w(z ; X)<1\right)>0$. Then we can find a compact subset $K$ of $\Delta$ contained in $X$ such that $\lim _{z \rightarrow \zeta} w(z ; X)<1$ on $K$ and $\mu(K)>0$. As

$$
1 \geqq w(z ; X) \geqq w(z ; K) \geqq 0,
$$

so by Lemma 3.4 , we see that $\lim _{z \rightarrow \zeta} w(z ; X)=1$ almost everywhere on $K$. This is a contradiction. Thus $\varlimsup_{z \rightarrow \zeta} w(z ; X)=1$ almost everywhere on $X$.

Considering $w(z ; \Delta-K)=1-w(z ; X)$, we get the second assertion of the theorem. This completes the proof.

Theorem 3.5. The harmonic measure $w(z ; X)$ is in the class $H D(R)$ if and only if there exists a compact subset $K$ of $\Delta$ such that

$$
\mu(X \cup K-X \cap K)=0 .
$$

Proof. The sufficiency of our condition is a direct consequence of Lemma 3.4. So we have only to prove the necessity of our condition.

Suppose that $w(z ; X)$ belongs to $H D(R)$. By Lemma 3.3, we can find a function $f(\zeta)$ in $U(\Delta)$ such that

$$
w(z ; X)=\int_{\Delta} f(\zeta) K(\zeta, z) d \mu(\zeta),
$$

5) Abreviation of "almost everywhere on $X$ ", i.e. "on $X$ except a set of $\mu$-measure zero". 
By Theorem 3.4, $\varlimsup_{z \rightarrow \zeta} w(z ; X)=1$ a.e. on $X$. By Theorem 3.3, $\lim _{z \rightarrow \zeta} w(z ; X)$ $=f(\zeta)$ on $\Delta$ except a set of measure zero. Hence we see that

$$
f(\zeta)=1
$$

a.e. on $X$. Let $K=\{\zeta ; \zeta$ is in $\Delta$ and $f(\zeta) \geqq 1\}$. As $f(\zeta)$ is upper semi-continuous, so $K$ is a compact subset of $\Delta$. Let $A=\{\zeta ; \zeta$ is in $X$ and $f(\zeta)=1\}$. Then $\mu(X-A)=0$ and $A$ is contained in $K$ and so $w(z ; X)=w(z ; A) \leqq w(z ; K)$ $\leqq \int_{K} f(\zeta) K(\zeta, z) d \mu(\zeta) \leqq w(z ; X)$. Hence $w(z ; K)=w(z ; A)$ and so $\mu(K-A)$ $=0$. Thus we obtain $\mu(X \cup K-X \cap K)=0$.

This completes the proof of Theorem 3.5.

6. HD-minimal functions. Corresponding to Theorem 3.2, we can prove the following measure theoretic characterization of $H D$-minimality:

Theorem 3.6. If $v(z)$ is HD-minimal, then there exists a point $\zeta_{0}$ in $\Delta$ with positive measure and a positive number $c$ such that

$$
v(z)=c w\left(z ; \zeta_{0}\right) \text {. }
$$

Conversely, if $\zeta_{0}$ is a point in $\Delta$ with positive measure, then $w\left(z ; \zeta_{0}\right)$ is $H D$ minimal.

Proof. Suppose that $v(z)$ is $H D$-minimal. By Corollary 2 of Theorem 3.4, $v(z)=\lim (v \wedge n)(z)$ on $R$. So some $(v \wedge n)(z)$ is not identically zero on $R$ and $v(z) \geqq(v \wedge n)(z)$. By the minimality of $v(z)$, we can find a positive number $c$ such that $v(z)=c(v \wedge n)(z)$. Thus we may assume that $\sup _{R} v(z)=1$.

Let $\bar{v}(\zeta)=\lim _{z \rightarrow \zeta} v(z)$ on $\Delta$. Then $\bar{v}(\zeta)$ is in $U(\Delta, \mu)$. By Theorem 3.3, we can write

$$
v(z)=\int_{\Delta} \bar{v}(\zeta) K(\zeta, z) d \mu(\zeta)
$$

Now we set $K_{n}=\{\zeta ; \zeta$ is in $\Delta$ and $\bar{v}(\zeta) \geqq 1 / n\}$. Then $K_{n}$ is a compact subset of $\Delta$ and $K_{n} \subset K_{n+1}$. As $n v(z) \geqq w\left(z ; K_{n}\right)$, so we can find a non negative number $c_{n}$ such that $w\left(z ; K_{n}\right)=c_{n} v(z)$. By Maximum principle, the set $\{\zeta$; $\zeta$ is in $\Delta$ and $\tilde{v}(\zeta)>0\}=\bigcup_{n=1}^{\infty} K_{n}$ is of positive measure. Hence we can find a number $n$ such that $\mu\left(K_{n}\right)>0$ and so $c_{n}>0$. By Theorem 3.4, $\sup _{n} w\left(z ; K_{n}\right)$ $=1$. So $c_{n}=1$ and $v(z)=w\left(z ; K_{n}\right)$. For simplicity, we put $K=K_{n}$. Then we obtain 


$$
v(z)=\int_{K} K(\zeta, z) d \mu(\zeta) .
$$

Next we show that $K$ is atomic, i.e. there exists no decomposition such that $K=A+B, A \cap B=$ empty and $\mu(A) \cdot \mu(B)>0$. Contrary to the assertion, assume that $K=A+B, A \cap B=$ empty and $\mu(A) \cdot \mu(B)>0$. Choose two compact sets $E$ and $F$ in $\Delta$ such that $E$ and $F$ are contained in $A$ and $B$ respectively and $\mu(E) \cdot \mu(F)>0$. Then

$$
v(z) \geqq w(z ; E) \quad \text { and } \quad w(z ; F) .
$$

Hence we can find a positive numbers $e$ and $f$ such that

$$
v(z)=e w(z ; E)=f w(z ; F) .
$$

By Theorem 3.4, we see that $e=f=1$. Hence $w(z ; E)=w(z ; F)$. As $F$ is contained in $\Delta-E$, so by Lemma $3.4, w(z ; E)$ vanishes continuously on $F$. Thus $w(z ; F)$ vanishes continuously on $F$. Also by Lemma $3.4, w(z ; F)$ vanishes continuously on $\Delta-F$. Hence $w(z ; F)$ vanishes continuously on $\Delta$. Thus by the maximum principle, $w(z ; F)$ is identically zero on $R$ or $\mu(F)=0$. This is absurd.

As $K$ is atomic, there exists a point $\zeta_{0}$ in $K$ of positive measure. In fact, suppose that every point $\zeta$ in $K$ has measure zero. Fix a point $\zeta$ in $K$. Then there exists a neighborhood $U=U(\zeta)$ of $\zeta$ such that $\mu(U \cap K)=0$. To see this, we choose a neighborhood $V$ of $\zeta$ such that $\mu(V)<\mu(K)$. Then $\mu(K)$ $=\mu(K \cap V)+\mu(K-K \cap V)$ implies $\mu(K \cap V)=\mu(K)$ or $\mu(K-K \cap V)=\mu(K)$ since $K$ is atomic. As $\mu(V)<\mu(K)$, so we must have $\mu(K \cap V)=0$. Thus we have only to take $U(\zeta)=V$. Now as $K$ is compact, so a finite number of such $U(\zeta)$ 's cover $K$, say $U\left(\zeta_{1}\right) \cap \cdots \cap U\left(\zeta_{n}\right) \supset K$. Then

$$
\mu(K) \leq \sum_{i=1}^{n} \mu\left(K \cap U\left(\zeta_{i}\right)\right)=0,
$$

which is a contradiction. Hence there exists a point $\zeta_{0}$ in $K$ such that $\mu\left(\zeta_{0}\right)>0$. Again by using the fact that $K$ is atomic, we get $\mu(K)=\mu\left(\zeta_{0}\right)$. Thus we find that

$$
v(z)=\int_{K} K(\zeta, z) d \mu(\zeta)=\int_{\zeta_{0}} K(\zeta . z) d_{\mu}(\zeta)=w\left(z ; \zeta_{0}\right)
$$

Conversely, suppose that $\zeta_{0}$ is a point in $\Delta$ such that $\mu\left(\zeta_{0}\right)>0$. If $w\left(z ; \zeta_{0}\right)$ $\geqq u(z)$ for some $u(z)$ in $H D(R)$, then 


$$
\lim _{z \rightarrow \zeta} w\left(z ; \zeta_{0}\right) \geqq \lim _{z \rightarrow \zeta} u(z)=\bar{u}(\zeta)
$$

on 4 . By Lemma 3.4

$$
\lim _{z \rightarrow \zeta} w\left(z ; \zeta_{0}\right)=\lim _{z \rightarrow \zeta} w\left(z ; \zeta_{0}\right)=0
$$

on $\Delta$ except at $\zeta_{0}$. Hence $\bar{u}(\zeta)=0$ on $\Delta$ except at $\zeta_{0}$. As

$$
u(z)=\int_{\Delta} \bar{u}(\zeta) K(\zeta, z) d \mu(\zeta)
$$

by Theorem 3.3 , so we get

$$
u(z)=\bar{u}\left(\zeta_{0}\right) \int_{\zeta_{0}} K(\zeta, z) d \mu(\zeta)=\bar{u}\left(\zeta_{0}\right) w\left(z ; \zeta_{0}\right) .
$$

Hence $w\left(z ; \zeta_{0}\right)$ is $H D$-minimal.

This completes the proof of Theorem 3.6.

\section{REFERENCES}

[1] N. Bourbaki: Topologie générale, Chap. X (1949).

[2] C. Constantinescu and A. Cornea: Über den idealen Rand und einige seiner Anwendungen bei der Klassification der Riemannschen Flächen, Nagoya Math. J., 13, 169-233 (1958).

[3] S. Kakutani: Concrete representation of abstract (M)-spaces, Ann. Math., 42, 9941024 (1941).

[4] S. Mori: A remark on a subdomain of a Riemann surface of the class $O_{H D}$, Proc. Japan Acad., 32, 251-254 (1958).

[5] S. Mori and M. Ôta: A remark on the ideal boundary of a Riemann surface, Proc. Japan Acad., 32, 409-411 (1956 .

[6] M. Nakai: On a ring isomorphism induced by quasiconformal mappings, Nagoya Math. J., 14, 201-221 (1959).

[7] M. Nakai: Algebraic criterion on quasiconformal equivalence of Riemann surfaces, Nagoya Math. J., 16, 157-184 (1960).

[ 8 ] H. L. Royden: Harmonic functions on open Riemann surface, Trans. Amer. Math. Soc., 73, 40-94 (1952).

[9] H. L. Royden: The ideal boundary of an open Riemann surface, Annals of Mathematics Studies, 30, 107-109 (1953).

[10] H. L. Royden: Open Riemann surfaces, Ann. Acad. Sci. Fenn., A.I. 249/5 (1958).

\section{Mathematical Institute}

Nagoya University 